\title{
Disruption of Robo2-Baiap2 integrated signaling drives cystic disease
}

\author{
Qinggang Li, ${ }^{1}$ Shaoyuan Cui, ${ }^{1}$ Qian Ma, ${ }^{1}$ Ying Liu, ${ }^{1}$ Hongyu Yu, ${ }^{1}$ GuangRui Geng, ${ }^{1}$ Ewud Agborbesong, ${ }^{2}$ \\ Chongyu Ren, ${ }^{3}$ Kai Wei, ${ }^{1}$ Yingjie Zhang, ${ }^{1}$ Jurong Yang, ${ }^{4}$ Xueyuan Bai, ${ }^{1}$ Guangyan Cai, ${ }^{1}$ \\ Yuansheng Xie, ${ }^{1}$ Xiaogang $\mathrm{Li}^{5}{ }^{5}$ and Xiangmei Chen ${ }^{1}$ \\ 'Department of Nephrology, Chinese PLA General Hospital, Medical School of Chinese PLA, Chinese PLA Institute of \\ Nephrology, State Key Laboratory of Kidney Diseases, National Clinical Research Center for Kidney Diseases, Beijing, \\ China. ${ }^{2}$ Department of Internal Medicine, Department of Biochemistry and Molecular Biology, University of Kansas Medical \\ Center, Kansas City, Kansas, USA. ${ }^{3}$ Department of Internal Medicine, University of Texas Southwestern Medical Center, \\ Dallas, Texas, USA. ${ }^{4}$ Department of Urology, Third Affiliated Hospital of Chongqing Medical University (General Hospital), \\ Chongqing, China. ${ }^{5}$ Department of Internal Medicine, Mayo Clinic, Rochester, Minnesota, USA.
}

Hereditary renal cystic diseases are characterized by defects in primary cilia of renal tubular epithelial cells and abnormality of tubular epithelium, which ultimately result in the development of renal cysts. However, the mechanism leading from abnormality of the tubular epithelium to cystogenesis is not well understood. In this report, we demonstrate a critical role for Robo2 in regulating epithelial development, including ciliogenesis, polarization, and differentiation. We found that Robo2 deficiency results in cystic kidneys, and the cyst cells showed defective cilia and polarity defects in tubular epithelium. The cyst cells, less than terminally differentiated, continue to proliferate. We further established that Robo2 works with $\mathrm{p} 53$ as well as polarity and ciliary proteins (Par3, PKCs, ZO-2, and Claudin-2) to regulate these processes. Robo2 binds to Baiap2 (also known as IRSp53) through the IRSp53/MIM homology domain in renal epithelial cells. This binding allows Robo2 to phosphorylate MDM2 at Ser166 via Baiap2 and maintain p53 homeostasis. Disruption of the Robo2-Baiap2 complex causes MDM2 to be subjected to dephosphorylation, leading to a high level of active $\mathrm{p} 53$, and initiated p53-mediated cellular

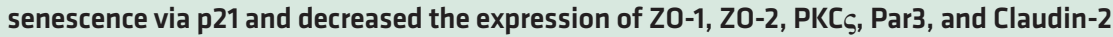
proteins, resulting in defects in epithelial development, including ciliogenesis, polarization, and differentiation. Importantly, double knockout of Robo2 and p53 rescued all the epithelial defects in kidneys compared with those in Robo2-knockout kidneys. Taken together, the present results demonstrate that Robo2 deficiency causes renal cystic disease, which is largely dependent on defective Robo2-Baiap2 integrated signaling in kidneys.

Conflict of interest: The authors have declared that no conflict of interest exists.

Copyright: (c) 2019, American Society for Clinical Investigation.

Submitted: January 24, 2019

Accepted: August 21, 2019

Published: September 19, 2019.

Reference information: /CI Insight. 2019;4(18):e127602. https://doi.org/10.1172/jici. insight.127602.

\section{Introduction}

Renal cystic diseases include autosomal dominant polycystic kidney disease, autosomal recessive $\mathrm{PKD}$, and others $(1,2)$. Moreover, most cysts are induced by the disruption of primary cilia and polarity $(1,2)$. Numerous kidney cysts accompany these polarity defects (3). Aberrant polarization of tubule cells results in inappropriate location of $\mathrm{Na}^{+} / \mathrm{K}^{+}$channels to the apical domain of the cells, resulting in dilation of tubule lumens. The proper polarization and differentiation of renal tubule epithelial cells are essential for kidney development that prevents abnormal cystic dilation.

The fundamental process of cellular differentiation polarization is established early during development (4). Multiple proteins and pathways have been identified as regulators of epithelial development throughout the polarization process. The apical-basal polarity of cells is a prerequisite for all aspects of epithelial cell function and is required for the proper localization of primary cilia (5), and cilia are required for maintaining cell polarity (5). The primary cilia of renal tubular epithelial cells also function to maintain normal tubular architecture, and dysfunction of primary cilia alters renal tubular cell proliferation and differentiation. However, interplay between the many pathways for epithelial development and function is still unclear.

Robo2, the cell surface receptor for the repulsive guidance cue Slit, is involved in axon guidance and neuronal migration $(6,7)$. The importance of Robo 2 signaling in metanephric kidney development 
A

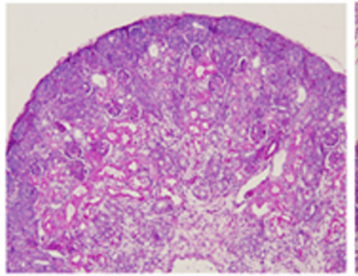

WT

C

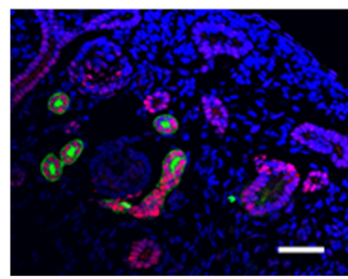

WT

E

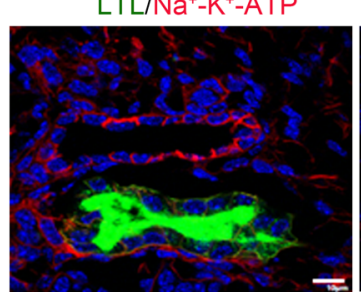

WT

G

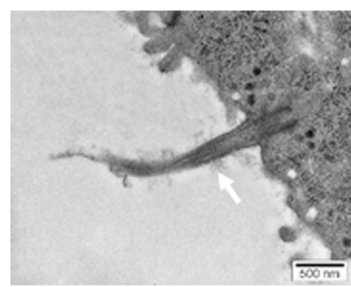

WT

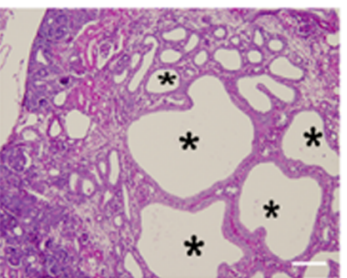

Robo2/-

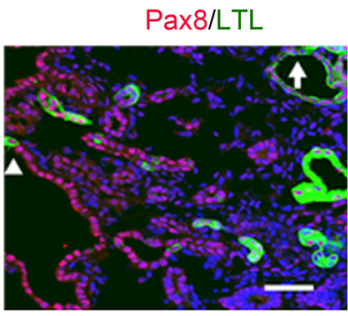

Robo2/-

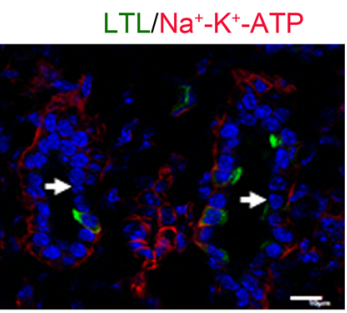

Robo2'

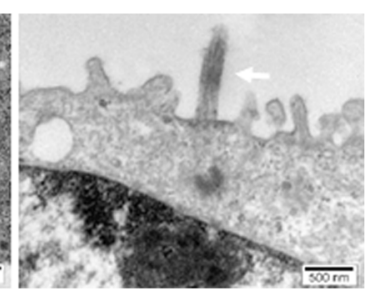

Robo2
B

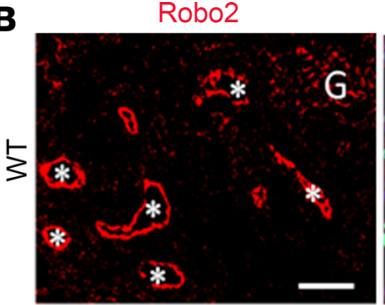

D

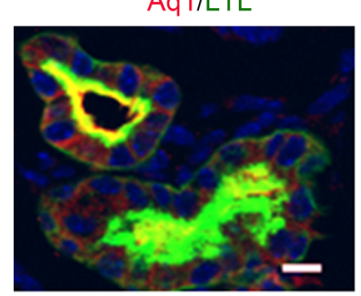

WT

$F$

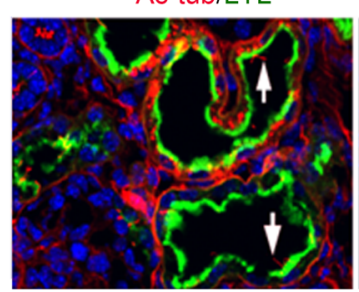

WT

H

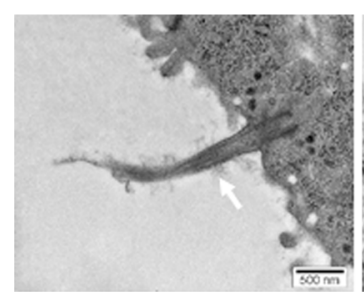

WT
Robo2/Pax8/DAPI

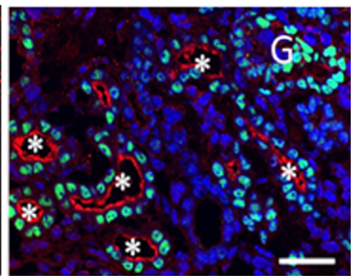

Aq1/LTL

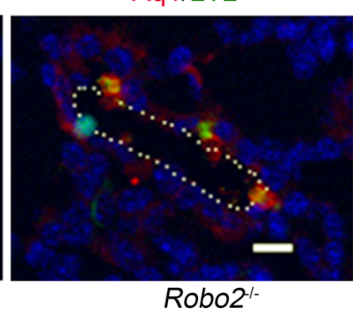

Ac-tub/LTL

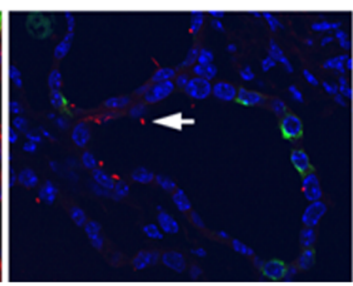

Robo2/

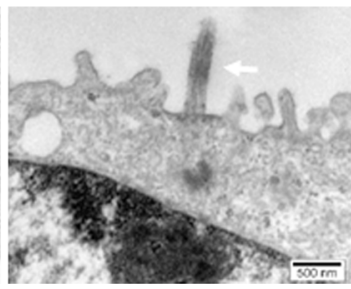

Robo2'

Figure 1. Robo2 is required for progenitor cells to differentiate into ciliated and polarized epithelium. (A) Representative H\&E-stained image

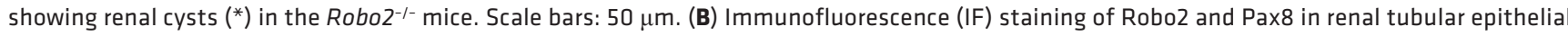
cells in E15.5 wild-type kidneys $(n=5)$. Scale bars: $50 \mu \mathrm{m}$. (C) IF staining of Pax8 and Lotus tetragonolobus lectin (LTL) in renal tubular epithelial cells in E17 wild-type and Robo2-knockout kidneys. Pax8 was expressed in cystic segments with reduced or absent LTL staining (white arrow and arrowhead). The representative image shows cysts $\left(^{*}\right)$ in the kidney. Scale bars: $50 \mu m$. (D) IF staining of Aqp1 and LTL in the cryosections of E17 wild-

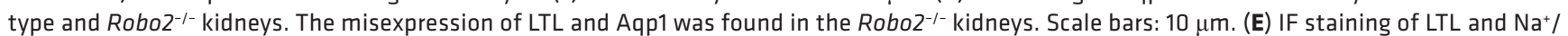
$\mathrm{K}^{+}$-ATPase in the Robo2 ${ }^{-1-}$ kidneys showed a lack of polarity in the renal tubule epithelial cells. Scale bars: $10 \mu \mathrm{m}$. Biological replicates: $n=10$. (F) IF staining of $\alpha$-tubulin and LTL in the cryosections of kidneys showed the defects of ciliogenesis in the Robo2 ${ }^{-1-}$ kidneys ( $\left.n=7\right)$. Scale bars: $10 \mu m$. (G) Transmission electron micrographs show that primary cilia (white arrow) defects (white arrowhead) occurred in the Robo2-/- kidney. Scale bars: 200

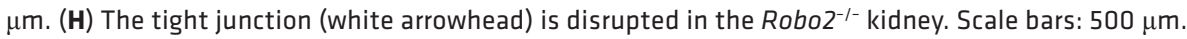

and renal tubular formation is underlined by the knockout mouse model for Robo2 as well as heterozygous $\mathrm{ROBO} 2$ mutations in humans with congenital anomalies of the kidney and urinary tract (8-12). We found that Robo2 deficiency results in cystic kidneys, and the cyst cells in the kidneys showed defective cilia and polarity defects in tubular epithelium. The link between abnormality of the tubular epithelium and cystogenesis remains elusive. We hypothesized that Robo2 plays a critical role in regulating epithelial development, including ciliogenesis, polarization, and differentiation. To address these unanswered questions, mouse models with selective deletion of Robo2 or p53 alleles were used for the following investigations. 


\section{Results}

Robo2 is required for progenitors to differentiate into renal tubular epithelial cells. Knockout of Robo2 resulted in defects in the Robo2 $2^{-1}$ kidneys (Figure 1A). To investigate whether Robo2 is involved in epithelial differentiation, we stained the E17 wild-type kidney sections and found that Robo2 was located in embryonic renal tubular cells (Figure 1B, left) and coexpressed with Pax8 (13-15) (an early proximal tubule marker) in these cells (Figure 1B). We further found that the abnormal epithelial cells from Robo2 ${ }^{-1-}$ kidneys stained positive for Pax8 (Figure 1C), Cited1 (a nephron progenitor marker) (refs. 16, 17; and Supplemental Figure 1B; supplemental material available online with this article; https://doi.org/10.1172/jci.insight.127602DS1), Sox9 (Sox $9^{+}$cells are progenitors in the kidney) (refs. 18-21 and Supplemental Figure 1C), and Aqp1 (a proximal tubule apical marker) (Figure 1D), whereas the staining of LTL (a proximal tubule apical marker) in these cells was weak, undetectable, or mislocalized (Figure 1, C-E, and G) compared with that in wild-type kidneys (22). In addition, the staining of $\mathrm{Na}^{+} / \mathrm{K}^{+}$-ATPase, a cell polarity marker, was markedly

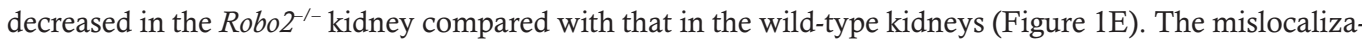
tion of LTL and $\mathrm{Na}^{+} / \mathrm{K}^{+}$-ATPase on the apical membranes suggested a lack of polarization and terminal

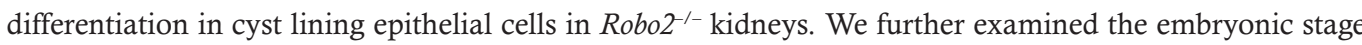
including E15.5 of mutant kidneys to show that failures in differentiation of renal progenitor cells into renal tubules occur from the beginning of nephrogenesis (Supplemental Figure 1A).

We also found that cilia were detected by the staining of $\alpha$-acetylated tubulin, a cilia marker, in tubular cells that were costained with LTL in wild-type kidneys (Figure 1F). However, knockout of Robo2 disrupted ciliogenesis (Figure 1G) and tight junction (Figure 1H) and resulted in short and dot-like cilia in cyst lining epithelial cells (Figure 1, F and G). We further found that knockout of Robo2 also resulted in short and dot-like cilia in mouse inner medullary collecting duct (IMCD3) cells (Supplemental Figure 2, A-C). These results suggested that the Robo2-deficient renal epithelial cells were incompletely differentiated and had defects in differentiation, including cell polarity and ciliogenesis.

Binding of Robo2 to Baiap2 forms a functional signaling complex. To understand the mechanism of how Robo2 regulates epithelial cell development/differentiation, polarization, and ciliogenesis, we identified the in vivo binding partners of Robo2 using coimmunoprecipitation (co-IP) (Supplemental Figure 3A) and mass spectrometric analysis (Supplemental Table 1). We found that Baiap2 (also known as Irsp53) might be the most likely candidate protein for Robo2 interaction.

We found that Baiap2 was colocalized with Robo2 and LTL basolaterally in embryonic epithelial cells in wild-type kidneys (Supplemental Figure 4, A and B). We confirmed the interactions between Robo2 and Baiap2 with His- and Myc-tagged human full-length Robo2 (His-myc-Robo2) or Flag-Baiap2 in HEK293T cells by co-IP assays (Figure 2A). Because Robo2's biological function depends upon the cc domain (Figure $2 \mathrm{~B}$ and ref. 23), we made several His- and Myc-tagged constructs of Robo2 with different cc domains and found that the crucial binding site of Robo2 with Baiap2 was located within amino acids 1301-1394 (CC3-1394, amino acids 1301-1394, which span from CC3 domain [amino acid 1301] to amino acid 1394) (Figure 2, C and D, and ref. 23). Last, we determined that Robo2 interacted with Baiap2 through the IMD (amino acids 1-250) but not the SH3 domain (amino acids 251-521) (Figure 2, D and E, and refs. 24-26) because His-myc-Robo2 (CC3-1394) could be co-IP only with Flag-Baiap2-IMD but not with Flag-Baiap2SH3 with Myc beads (Figure 2, D, E, and G). An in vivo co-IP experiment using kidney tissue lysates also showed that Robo2 interacted with Baiap2 in vivo (Figure $2 \mathrm{~F}$ ).

To rule out an indirect association of Robo2 and Baiap2 in a protein complex and demonstrate the direct interaction of Robo2 and Baiap2, we did a pull-down assay to prove the direct interaction of Robo2 and Baiap2 (Supplemental Figure 3, B-E).

To explore the functional role of the interaction between Robo2 and Baiap2, we coexpressed these proteins in Madin-Darby canine kidney (MDCK) cells. We found that Robo2 could recruit Baiap2 to the cell membrane in cells coexpressing Robo2 and Baiap2 (Supplemental Figure 4C), and 68\% of cells were positive for both Robo2-GFP and Flag-Baiap2. In contrast, the cells transfected with Baiap2 alone showed a diffuse punctate distribution of Baiap2 throughout the cytoplasm rather than membrane localization (Supplemental Figure 4C). Similar to the localization pattern of Baiap2 in vitro, we found that Baiap2 was accumulated at the tight junctions in the wild-type mouse kidneys and was almost undetectable in the Robo2 ${ }^{-1-}$ kidney (Supplemental Figure 4B). Previous reports demonstrated that Baiap2 is associated with epithelial differentiation and polarization (27); our results suggest that the localization of Baiap2 depends on the presence of Robo2, which may be required for cell differentiation. 
A

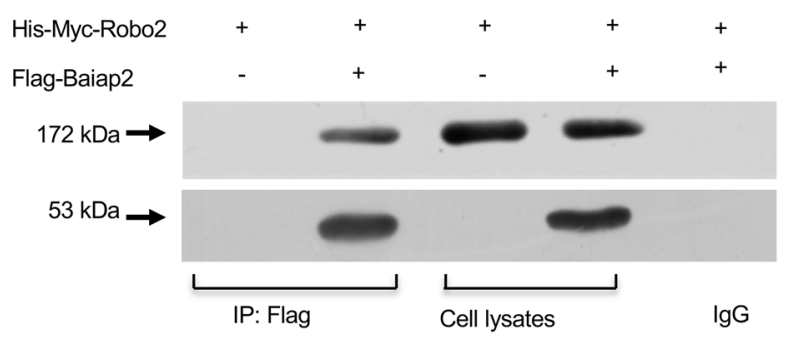

B

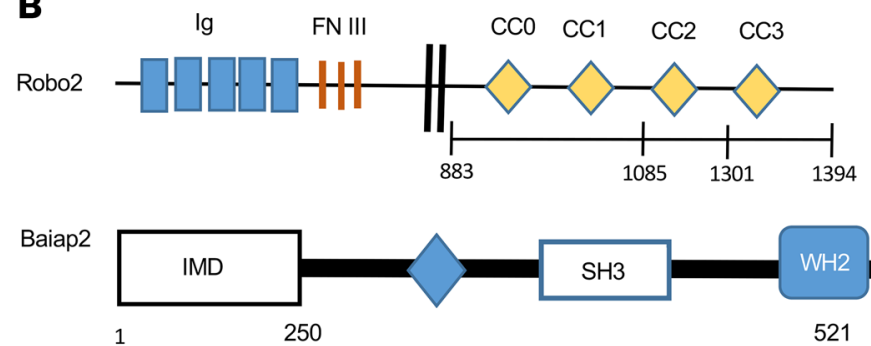

C

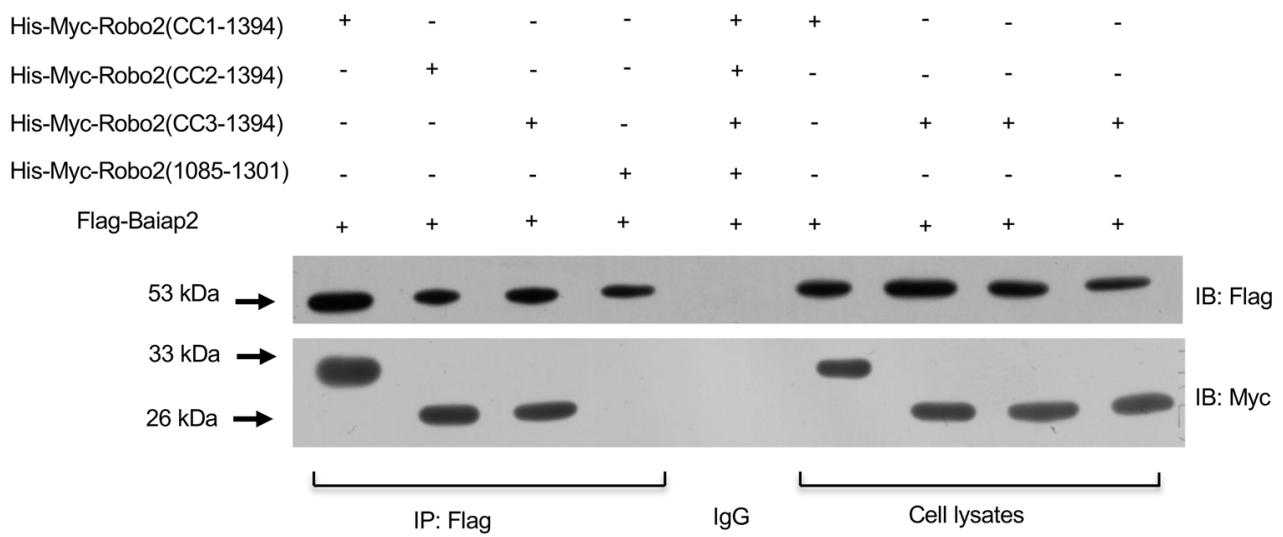

D

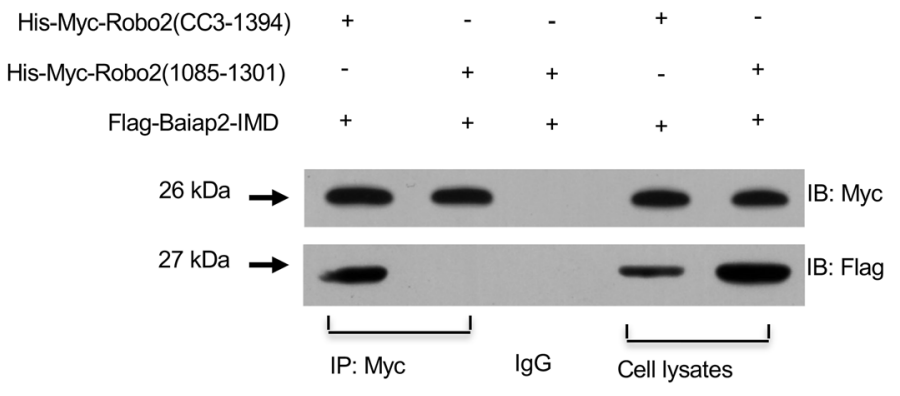

$\mathbf{F}$
E His-Myc-Robo2(CC3-1394) His-Myc-Robo2(1085-1301)

Flag-Baiap2(251-521)

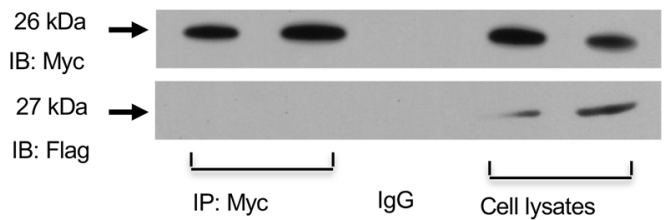

G

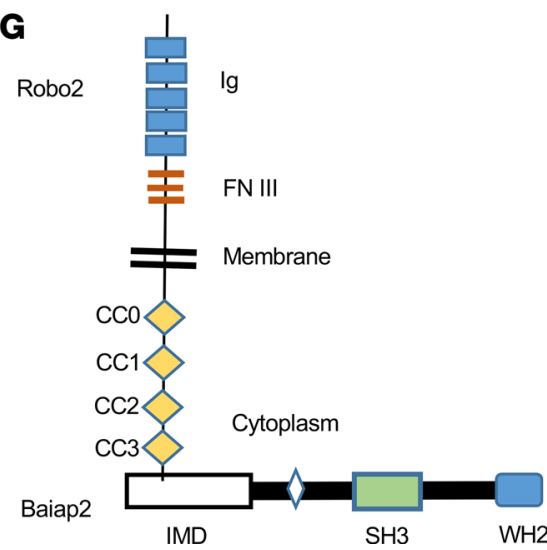

Figure 2. Robo2 directly interacts with Baiap2 via its IRSp53 and MIM domain. (A) Co-IP of Robo2 and Flag-tagged Baiap2 using the anti-Flag antibody. (B) Schematic diagram of the domain structure of the Robo2 and Baiap2 proteins. Ig, immunoglobulin domain; FN III, fibronectin type 3 domain; CC, cytoplasmic region; IMD, IRSp53/MIM homology domain (amino acids 1-250); SH3, Src homology 3 domain; WH2, WASP-homology 2 domain. The numbers indicate the positions in the full-length Robo2 or Baiap2 gene. (C) The co-IP assays showed that the region from CC3-1394 in Robo2 is required for its interaction with Baiap2. (D and $\mathbf{E}$ ) The co-IP assays showed that the IMD of Baiap2 is required for its interaction with the region from CC3-1394 in Robo2. (F) Co-IP analysis of endogenous Robo2 interacting with the exogenous Baiap2 using kidney tissue lysates. (G) The schematic diagram shows the binding domains of Robo2 and Baiap2. 
Robo2-Baiap2 acts as a negative regulator of p53-mediated cellular senescence. To further explore the roles of Robo2-Baiap2 integrated signaling in renal epithelial cells, we analyzed the gene expression profile in the Robo2 $2^{-1}$ E17.5 kidneys compared with that in wild-type kidneys using a microarray platform (Supplemental Table 2 and Figure 3A). We found that 240 genes were upregulated and 232 genes were downregulated upon Robo2 deletion ( $>1.2$-fold in either direction), which were then classified into several functional groups (Figure 3A, right). The Robo2-regulated genes and subgroups of different molecular pathways are also shown in Supplemental Table 2. We found that the most commonly upregulated and downregulated genes controlling cell cycle and cellular adhesion were also involved in the upregulation of p53 signaling proteins and the downregulation of differentiation genes. We selected and confirmed the expression of 9 genes, including Trp53 (p53), Cdkn1a (p21), Tjp1 (ZO-1), Cdkn1b, B-Raf, Cdc42, Ctnna1, Ctnnb1, and Pten by NanoString nCounter Gene Expression Assay (Figure 3B), which play important roles in mediating senescence and differentiation (28-32). The expression of p53 and p21 proteins was increased, and the expression of cyclin-dependent kinase $4(\mathrm{Cdk} 4)$ protein was decreased in Robo2 $2^{-1}$ kidneys compared with that in wild-type kidneys in vivo (Figure 3C).

We further found that knockdown of Robo2 not only increased the nuclear p53 in MDCK cells (Figure 4A) but also led to DNA damage in these cells as shown by $\gamma$-H2AX staining (a hallmark of DNA damage) (Figure 4B and ref. 33). Knockdown of Robo2 or Baiap2 also showed a high level of $\gamma$-H2AX by Western

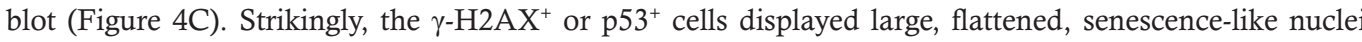
(Figure 4, A and B). Additionally, senescence-associated $\beta$-galactosidase (SA $\beta$-Gal) activity was increased

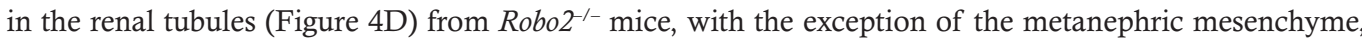
which is not observed in other models (34). Consistent with the elevated p53 and p21 levels, the Robo2 $2^{-1-}$ mice exhibited p21 expression in epithelial cells lacking differentiation and polarization (Supplemental Figure $5 \mathrm{~B}$ ). Taken together, these data suggest that loss of Robo2 could induce senescence, which might be through the unreported $\mathrm{p} 53 / \mathrm{p} 21$ pathway.

Because loss of Robo2 led to the upregulation of p53 and resulted in p53-mediated senescence (Figure 3, A-C; Supplemental Figure 6B; and ref. 29), and knockdown of Robo2 or Baiap2 also showed high level of $\gamma$-H2AX by Western blot (Figure 4C), we investigated the involvement of the Baiap2 cascade in $\mathrm{p} 53$ signaling. It has been reported that the phosphorylation of MDM2 promotes p53 degradation while the dephosphorylation of MDM2 can stabilize nuclear p53 $(35,36)$. We found that Robo 2 was required to maintain the phosphorylation of MDM2 at Ser166 because Robo2 deficiency led to the dephosphorylation of MDM2 in vitro and in vivo (Figure 4, E and F, and Supplemental Figure 6, A and B). We further found that Baiap2 deficiency also caused dephosphorylation of MDM2 at Ser166 (Figure 4G). These data indicate that Robo2 deficiency results in the dephosphorylation of MDM2 and subsequent phosphorylation of p53 through the Baiap2 cascade, ultimately resulting in the upregulation of p53. Further, knockdown of Robo2 or Baiap2 alone also resulted in upregulated expression of H2AX in MDCK cells (Figure 4C), which suggested that silence of Robo2/Baiap2 can contribute to DNA damage and even induce cellular senescence.

We further found that Slit2 has no significant direct effect on Baiap2, p53, and phosphorylated MDM2 after Robo2 knockdown (Supplemental Figure 6A), although Robo2 is the receptor for repulsive ligand Slit2. These data suggested that the effects of the Robo2-Baiap2 signaling pathway seem independent of Slit regulation on the Robo2 receptor.

Silence of Robo2 disrupts the normal program of cellular differentiation. Because ZO-1 is required for epithelial morphogenesis and lumen development (37-39), we further found that the expression of ZO-1 in renal

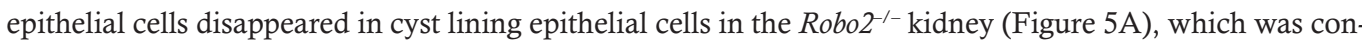
sistent with the downregulation of the ZO-1 encoding gene, Tjp1, in these mice (Figure 3B). Knockdown of Robo2 also dramatically decreased the expression of ZO-1 in MDCK cells (Figure 5, B-D). These results

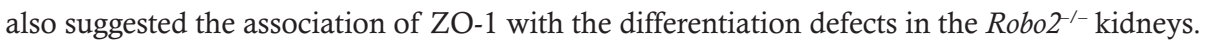

We further found that silence of Robo2 not only decreased the expression of ZO-1 in vivo and in vitro (Figure 3B, Figure 5, A-D, and Figure 6A) but also decreased the in vivo expression of PKC5, Par3, Claudin-2, and ZO-2 (Figure 5E), which are members of the ciliary and polarity protein complex $(37,40,41)$. The expression of ZO-1 could be found in Robo2 $2^{-1-}$ kidneys (Figure 6A and Supplemental Figure 6B). Collectively, our results suggest a requirement for the Robo 2 signaling cascade in progenitor differentiation. Moreover, because $\mathrm{PKC} \zeta$, Par3, and Claudin-2 are also ciliary proteins $(5,41)$, it is possible that these proteins might also be involved in

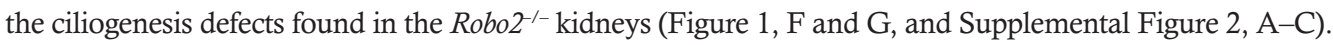




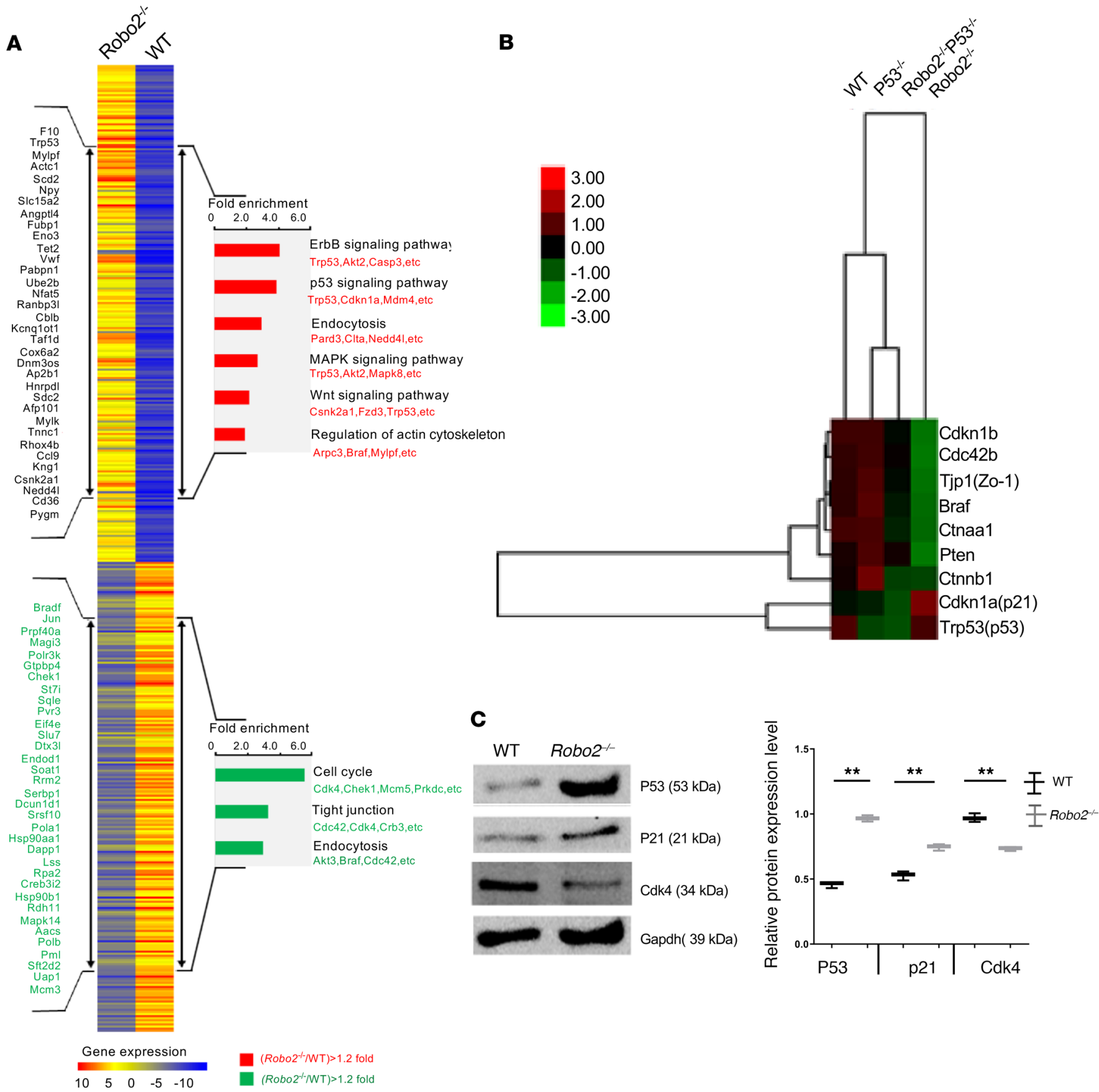

Figure 3. Knockout of Robo2 changes the transcription of genes associated with different signaling pathways in Robo2 ${ }^{-1-}$ kidneys compared with those in wild-type kidneys. (A) The gene expression profiles between the E17 wild-type and Robo2-1- mouse kidneys. The double-headed arrows indicate that 240 genes were upregulated and 232 genes were downregulated by Robo2. Some of the Robo2-repressed genes (black) and Robo2-induced genes (green) are listed vertically (left), and some of the potential Robo2-related molecular pathways are indicated on the right. (B) NanoString nCounter Cene Expression Assay was used to

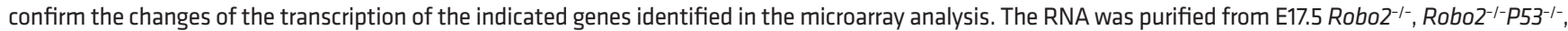
$\mathrm{P} 3^{-1-}$, and wild-type kidneys. The relative expression is shown as the fold difference relative to Gapdh, Actb, and B2m. (C) The expression of p53 and p21 proteins was increased, and the expression of CDK4 proteins was decreased in the Robo2 ${ }^{-1-}$ kidneys compared with those in wild-type kidneys. Data are mean \pm SEM. ${ }^{* *} P<0.01$ by 2 -tailed Student's $t$ test.

Robo2 mutations result in the abnormality of tubular epithelium because of p53-mediated signaling. To investigate the relationship between cell differentiation and p53-mediated senescence and determine whether the phenotypes

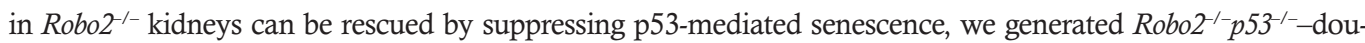
ble knockout mice. We found that double knockout of Robo2 and p53 restored the expression of Cdkn1a (p21) as examined by the NanoString assay analysis (Figure 3B) as well as the expression of P21, CDK4, PKC Claudin-2, and ZO-2 in kidneys compared with their expression in the Robo2 ${ }^{-1-}$ mouse kidneys (Figure 6A). 
A

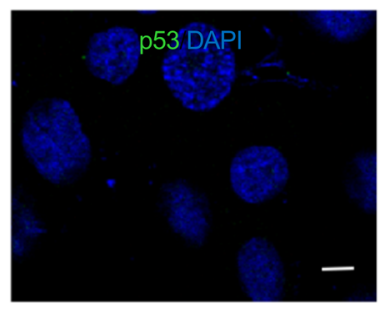

C
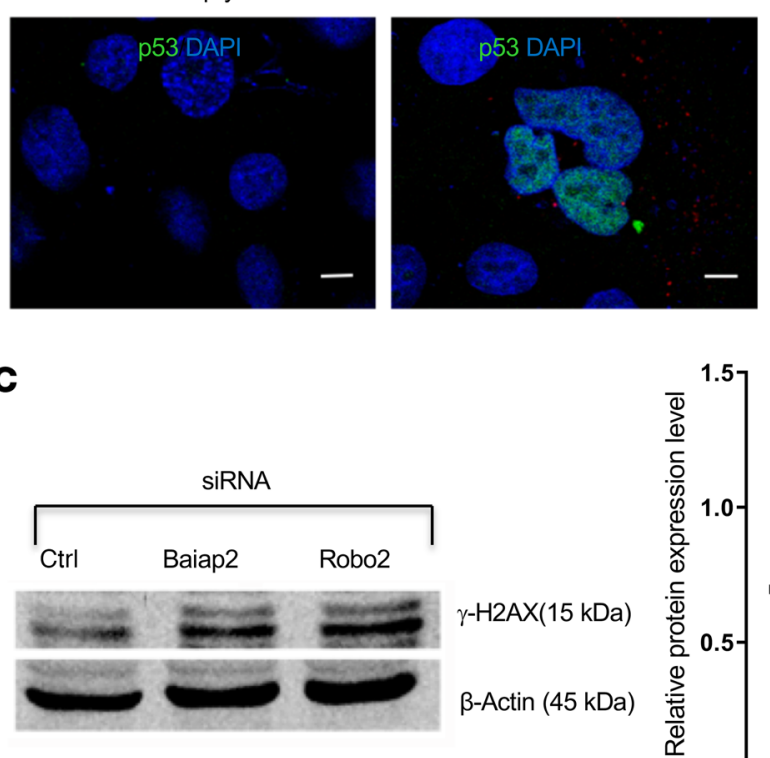

B

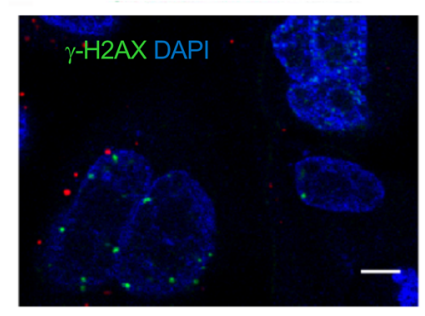

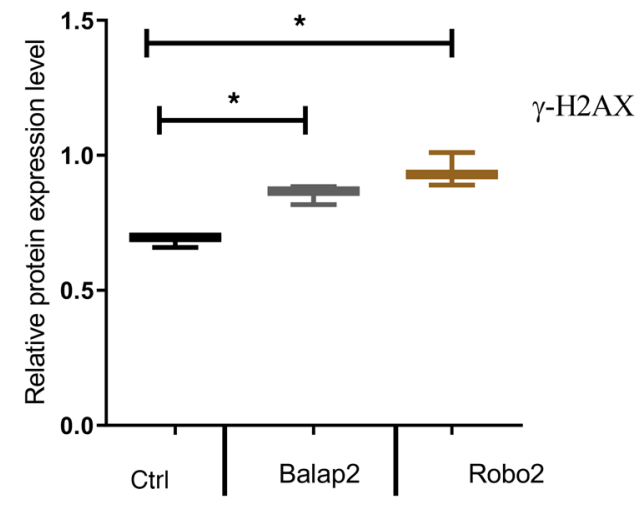

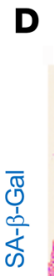

WT

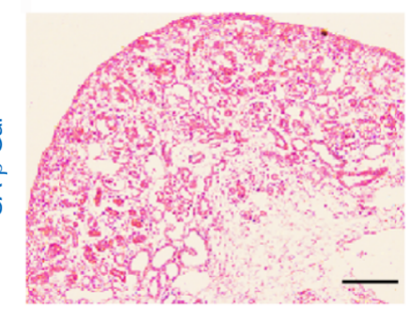

$\mathbf{F}$
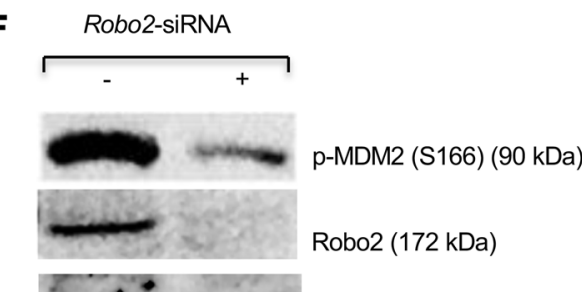

P-p53(S9) (53 kDa)

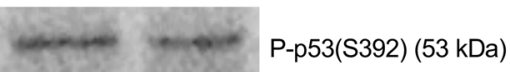

Gapdh (37 kDa)

G

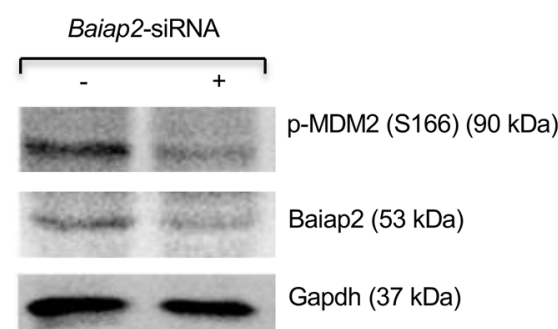

E
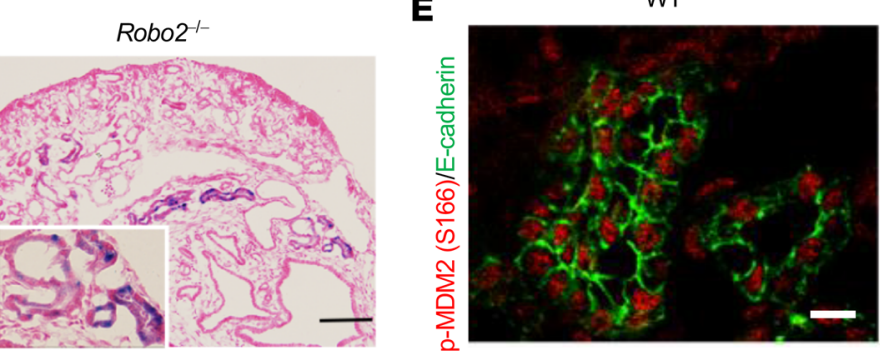

Robo2-1-
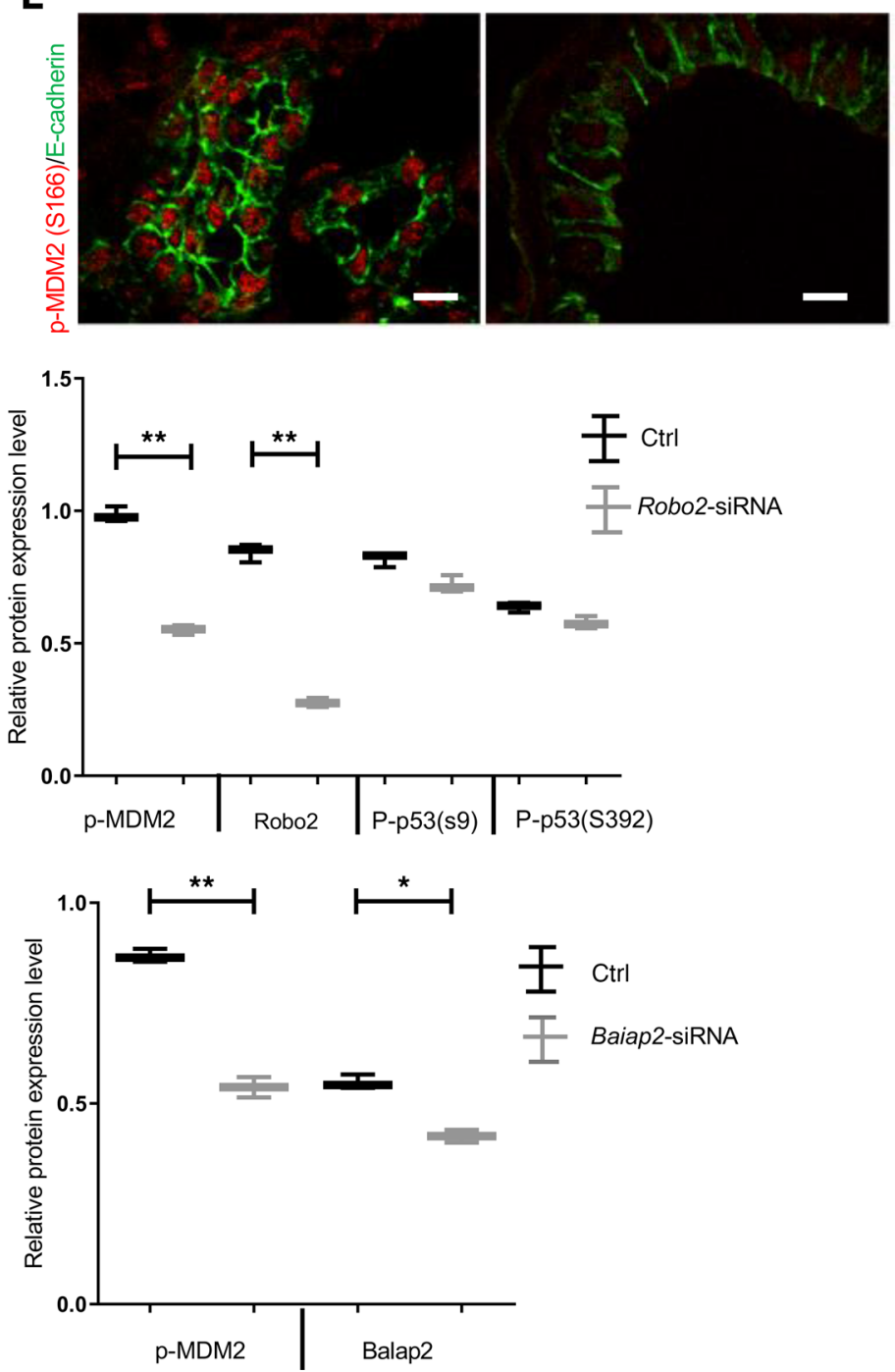
Figure 4. Disruption of Robo2-Baiap2 signaling results in p53-mediated cellular senescence. (A) IF staining of p53 in Robo2-knockdown MDCK cells. Scale bars: $20 \mu \mathrm{m}$. (B) IF staining of $\gamma$-H2AX in Robo2-knockdown MDCK cells. The red dots just show the labeling of siRNA with Cy3 (Robo2 siRNA). Scale bars: $20 \mu \mathrm{m}$. (C) Knockdown of Baiap2 or Robo2 increased the expression of $\gamma-\mathrm{H} 2 \mathrm{AX}$ in MDCK cells. Data are mean $\pm \mathrm{SEM}$. ${ }^{*} P<0.05$ compared with control group, 1-way ANOVA with Dunnett's post hoc test. (D) Staining of SA $\beta$-Gal in E17 wild-type and Robo2 ${ }^{-1-}$ kidneys. Scale bars: $50 \mu \mathrm{m}$. Original magnification, $\times 400$ (inset). (E) Knockout of Robo2 decreased the phosphorylation of MDM2 at Ser166 in Robo2 ${ }^{-1-}$ kidneys. Scale bars: $10 \mu \mathrm{m}$. (F) Knockdown of Robo2 decreased the phosphorylation of MDM2 at Ser166 in MDCK cells. Data are mean \pm SEM. ${ }^{* *} P<0.01$ by 2-tailed Student's $t$ test. (G) Knockdown of Baiap2 also decreased the phosphorylation of MDM2 at Ser166. Data are mean \pm SEM. ${ }^{*} P<0.05 ;{ }^{* *} P<0.01$ by 2 -tailed Student's $t$ test.

Moreover, we found that double knockout of Robo2 and $p 53$ prevented renal cyst formation (Figure $6, \mathrm{~B}, \mathrm{C}$, and F) and normalized the cell polarization defects compared with those in the Robo2 $2^{-1-}$ mouse kidneys (Figure 6D). In addition, the SA $\beta-G a l$ staining in the renal tubule cells was absent in

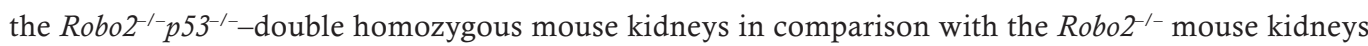
(Figure 6, E and G). These findings indicate that Robo2 mutations result in differentiation defects because of p53-mediated progenitor cell senescence (Figure 7).

\section{Discussion}

Renal cystic diseases encompass a wide spectrum of inherited, developmental, and acquired disorders $(1,2)$, and the hereditary renal cystic diseases are caused by abnormality of tubular epithelium, including defects in differentiation, polarization, and ciliogenesis. In this study, we found that Robo2 deficiency results in cystic kidneys and described the potentially novel roles and mechanisms of Robo2 in regulating renal tubular epithelial cell development. We found that deletion of Robo2 induced renal epithelial defects on progenitor differentiation, polarization, and ciliogenesis in vivo. We successfully showed that Robo2 regulates these processes through Baiap2-mediated p53 as well as ciliary and polarity proteins Par3, PKC, ZO-2, and Claudin-2.

Baiap2 is a multidomain protein that is able to associate with the Rho-GTPases. Rac is required for epithelial differentiation, mainly through its amino-terminal IMD $(27,42)$. We found that Baiap2 bound to Robo2 via this IMD (Figure 2G), which suggested that these proteins could form a protein complex. Our data indicated that total p53 protein can be upregulated after Robo2 knockout (Figure 3C and Supplemental Figure 6B) or Robo2 siRNA knockdown (Supplemental Figure 6A), although the phosphorylation of p53 was not affected (Figure 4F). MDM2 is well known as an ubiquitin E3 ligase that promotes p53 degradation (43). The stability of MDM2 can be regulated by phosphorylation at Ser166, making it a crucial regulatory residue. Akt-mediated phosphorylation of MDM2 at Ser166 results in MDM2-mediated ubiquitination and degradation of p53 $(44,45)$. Additionally, MDM2 has a role in abrogating the activity of p53 to induce growth arrest and apoptosis. Importantly, the interaction between p53 and MDM2 can be modulated at sites on both molecules, so the regulation of MDM2 is equally important in the p53 responses. We found that Robo2 and Baiap2 were required for maintaining the phosphorylation of MDM2 at Ser166 because Robo2 or Baiap2 deficiency led to the dephosphorylation of MDM2 and the upregulation of p53 (Figure 4, F and G; Supplemental Figure 6, A and B; and Figure 7). Abnormally high levels of wild-type p53 in the ureteric bud appear to alter cellular differentiation in embryonic kidney and cause inefficient conversion to epithelium in the adjacent undifferentiated mesenchyme (46). The $U B^{\text {mdm2-/- }}$ mutant phenotype is mediated by aberrant p53 activity (47), which is similar to the Robo2-deficient phenotype in our study. Our previous experiment on cysts derived from Pkd1-mutant renal epithelial cells showed deacetylation of p53 (48), which indicated that the level of p53 was crucial for renal cyst formation. Thus, our results provide the first demonstration to our knowledge of an important role played by Robo2 in the modulation of p53 activity in renal tubules and stability via regulating the phosphorylation of MDM2, which further supports that MDM2-mediated inhibition of p53 activity is a prerequisite for renal organogenesis.

Multiple observations led to the hypothesis that the senescent cells do not reach terminal differentiation. Similar to the depolarized and dedifferentiated epithelial cells in cystic renal disease, the Robo2-deficient epithelial cells also displayed arrested differentiation and polarization. We found that the Robo2 $2^{--}$epithelial cells positive for Pax8 (Figure 2B), Cited1 (Supplemental Figure 1B), Sox9 (Supplemental Figure 1C), and E-cadherin staining (Supplemental Figure 5A) remained as progenitor

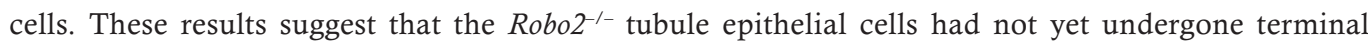
differentiation. Robo2 colocalized with tight junction protein ZO-1 and Claudin-2 (Supplemental Figure 4, D and E), and works together with polarity proteins Par3, Par6, and PKC $(37,40,41)$ as well, which are required for epithelial polarization (49). In this study, we found that Robo2 deficiency results 
A
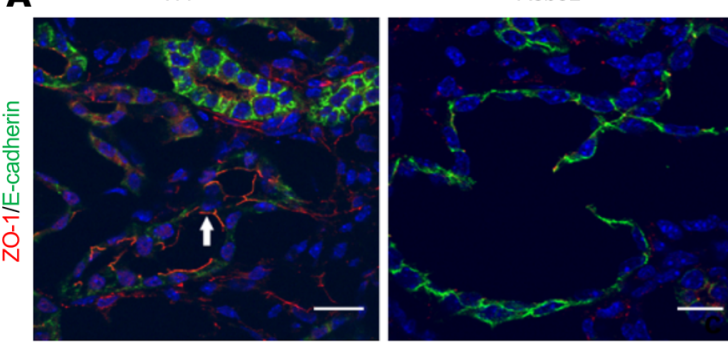

B

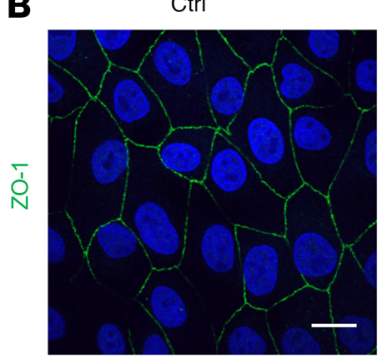

D Robo2-siRNA
C $\mathrm{Ctrl}$

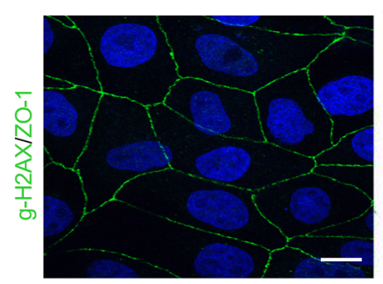

Robo2-siRNA

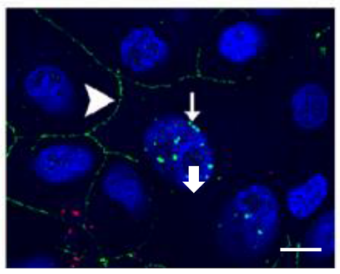

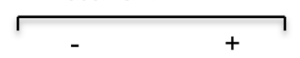
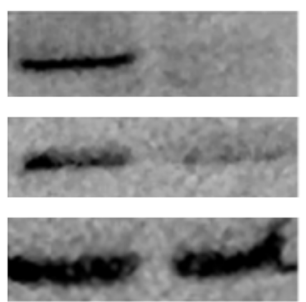

Robo2 (172 kDa)

ZO-1 (220 kDa)

Actin $(45 \mathrm{kDa})$

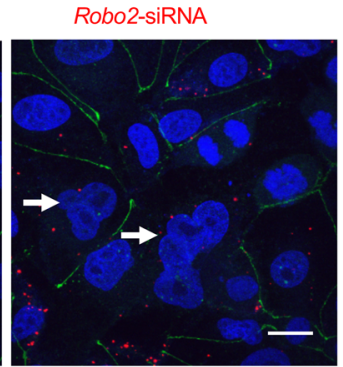

E
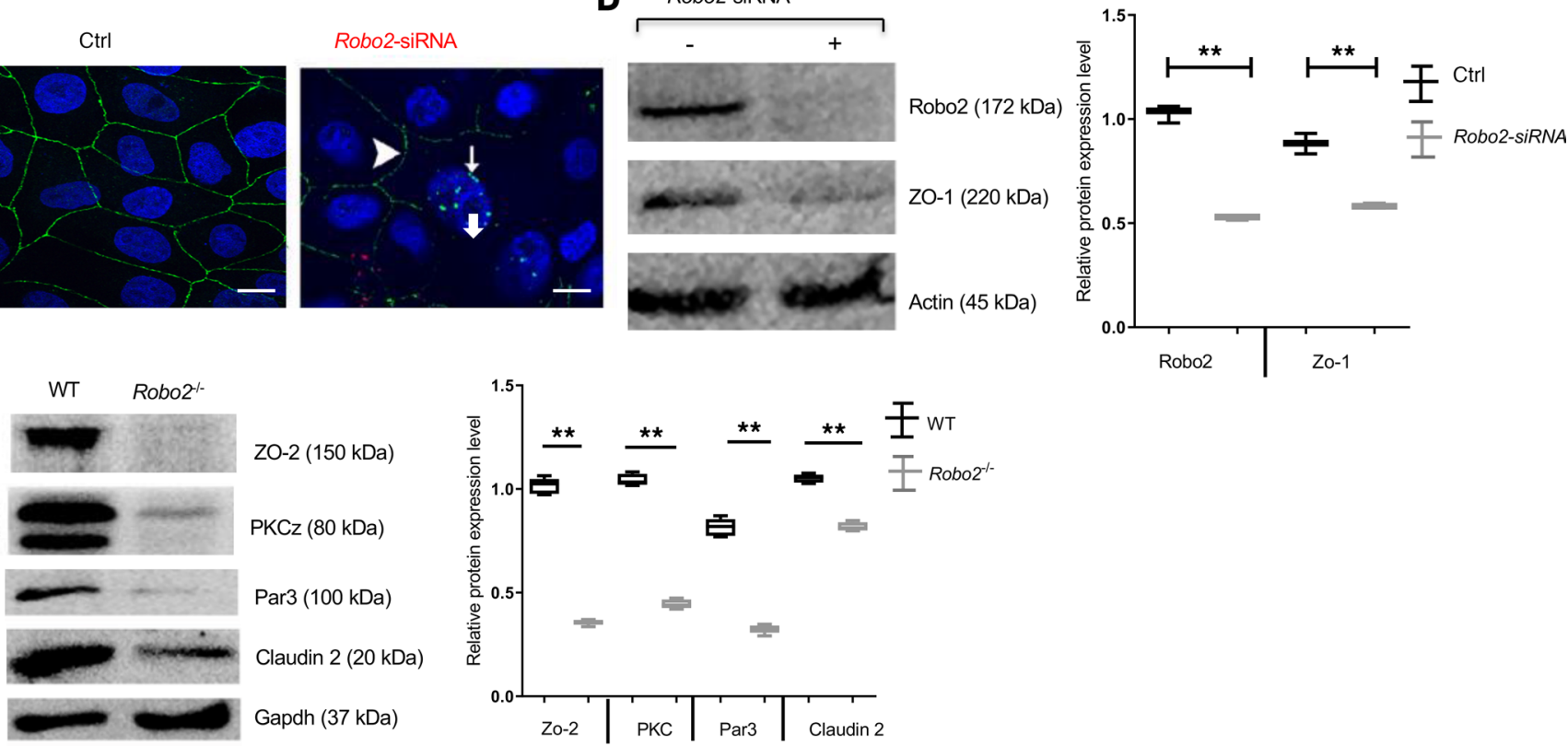

Figure 5. Silence of Robo2 signaling disrupts the normal program of cellular differentiation. (A) ZO-1 disappeared from cyst lining epithelial cells in the Robo2-1- kidneys. Scale bars: $10 \mu \mathrm{m}$. (B) Knockdown of Robo2 decreased Z0-1 expression in the MDCK cells and induced senescence-like cells (arrow). The red dots also show the labeling of siRNA with Cy3 (Robo2 siRNA), but the control siRNA was not labeled. Scale bars: $10 \mu \mathrm{m}$. (C) IF staining of $\gamma-\mathrm{H} 2 \mathrm{AX}$ and ZO-1 in Robo2-knockdown MDCK cells. Knockdown of Robo2 decreased Z0-1 expression in the MDCK cells and induced senescence (arrow). The red dots just show the labeling of siRNA with Cy3 (Robo2 siRNA). (D) Robo2 knockdown significantly deceased the ZO-1 protein level in the MDCK cells. Data are mean \pm SEM. ${ }^{* *} P<0.01$ by 2 -tailed Student's $t$ test. (E) The expression of the polarity proteins, including ZO-2, PKC $\zeta$, Par3, and Claudin-2, was decreased in Robo2 ${ }^{-/-}$kidneys compared with that in wild-type kidneys $(n=5)$. Data are mean \pm SEM. ${ }^{* *} P<0.01$ by 2 -tailed Student's $t$ test.

in decreased expression of ZO-1, Claudin-2, and Par3/Par6/PKC $\zeta$, finally contributing to abnormal tubule epithelial cell polarity in the mutants. These results demonstrate that Robo2, as a potentially novel tight junction protein, plays an essential role in the establishment and maintenance of apical-basal polarity during epithelial morphogenesis and lumen development.

The differentiation of tubular lining epithelial cells is essential for normal kidney development, and membrane protein polarity defects include the misplacement of normally basolateral membrane proteins to the apical surface and the failure to traffic and insert proteins into membranes, resulting in their intracellular accumulation. We found that polarity proteins Claudin-2 and Par3 were decreased in Robo2-deficient mouse kidneys and in Robo2-knockdown MDCK cells (Figure 5E). Par3 is required for the establishment of epithelial cell polarity (49). Par3 inactivation results in defective convergent extension and tubular morphogenesis as well as renal cyst formation (40). Claudin-2 is present at the cilium as a ciliary protein (41), which is also crucial to epithelial cell polarization $(37,50)$. The apical-basal polarity is also required for the proper localization of cilia (5). Our results suggest that Robo2-Baiap2 signaling may be required to recruit polarity and ciliary proteins, including Par3 and Claudin-2, to regulate epithelial differentiation, including polarization and ciliogenesis.

We further hypothesized that the abnormality of tubular epithelium was due to the abnormal activation of p53 (Figure 7). Appropriately, we found that a double knockout of Robo2 and $p 53$ not only rescued 


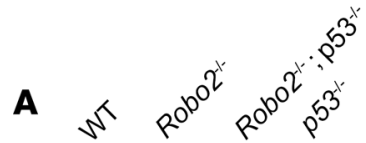

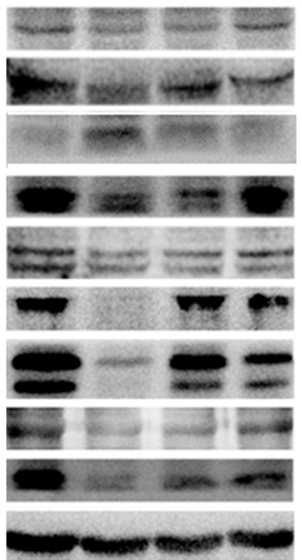

Robo2 (172 kDa)

Baiap2 (53 kDa)

P21 (21 kDa)

CDK4 (34 kDa)

ZO-1 (220 kDa)

ZO-2 (150 kDa)

$\mathrm{PKCz}(80 \mathrm{kDa})$

Par6 (43 kDa)

Claudin-2 (20 kDa)

Gapdh (37 kDa)

B

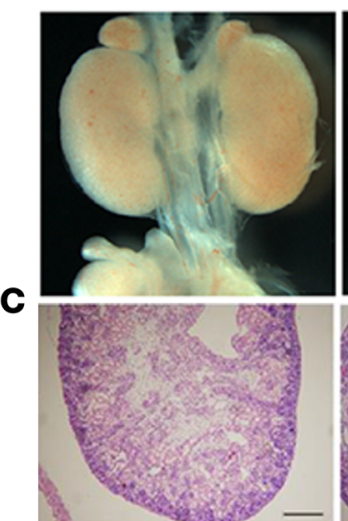

WT

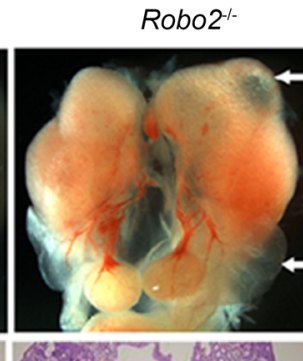

Robo2
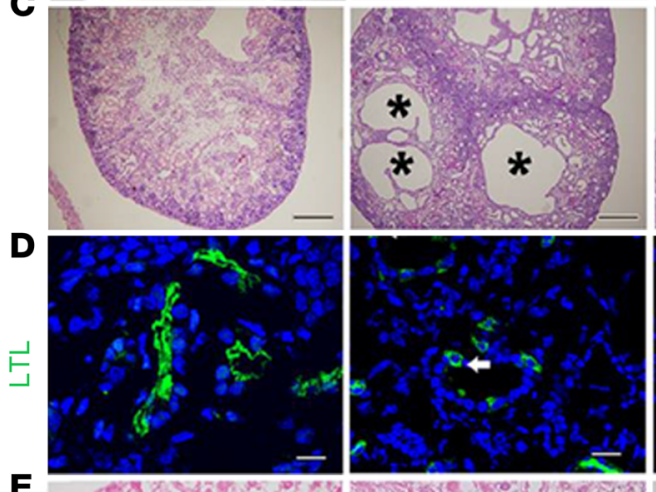

E
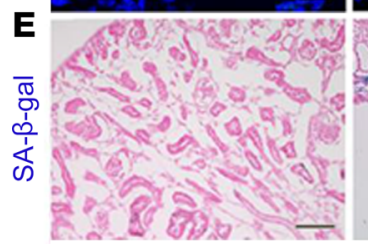

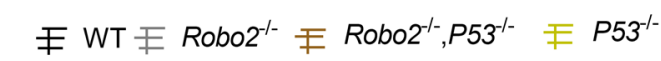

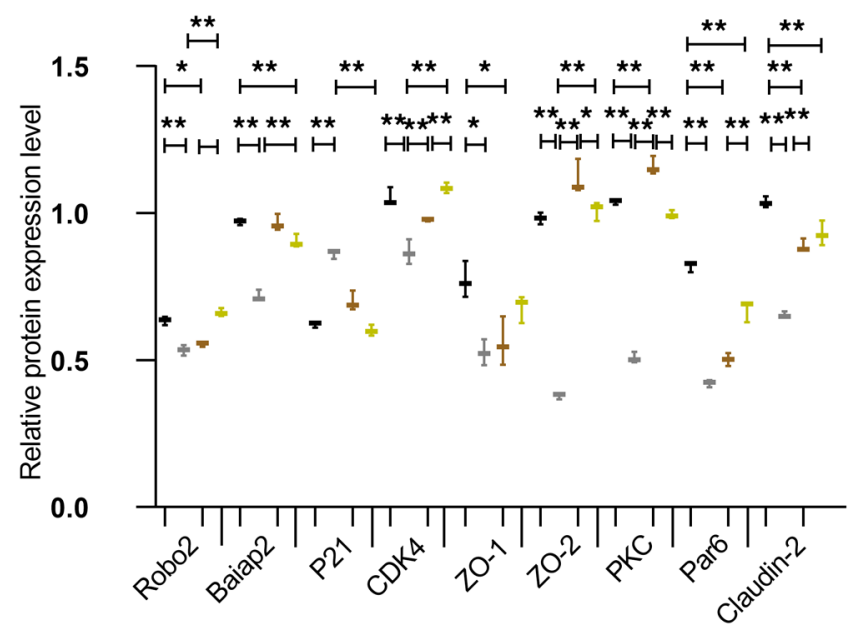

Robo2 ${ }^{-1} ; p 53^{-1}$

F
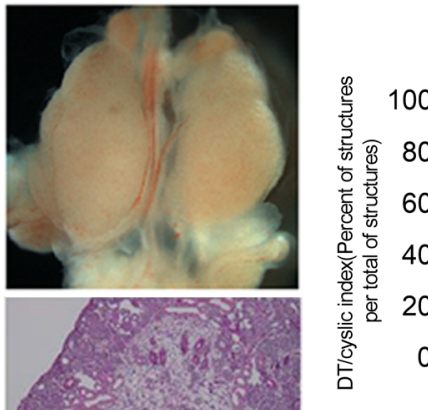

** *

$\stackrel{\star * \star *}{\mapsto}$

玉 WT

王 Robo2-1-

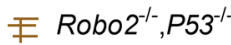
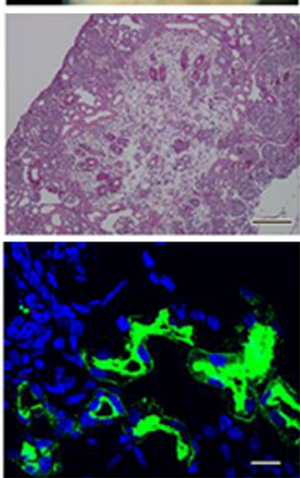

G

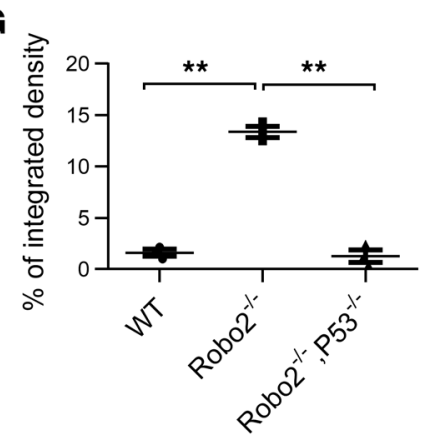

Figure 6. Double knockout of Robo2 and p53 rescues the defects observed in Robo2-single knockout kidneys. (A) Double knockout of Robo2 and p53 rescued expression of p21, ZO-1, ZO-2, PKC $\zeta$, and Claudin-2 $(n=3)$. Data are mean \pm SEM. ${ }^{*} P<0.05 ;{ }^{* *} P<0.01$ compared with wild-type group, 1-way ANOVA with Bonferroni's post hoc test. (B) Double knockout of Robo2 and $p 53$ rescued the Robo2-deficient kidney phenotype. No renal cysts could be found in the Robo2 $2^{-1-} p 53^{-/-}$kidney. White arrows indicate cysts. (C and F) Double knockout of Robo2 and p53 prevented cyst formation (see asterisks), which was observed in Robo2-deficient kidneys, and histological analysis revealed that the morphology of the renal tubules in the dou-

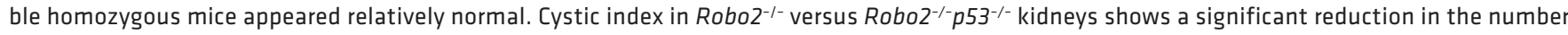

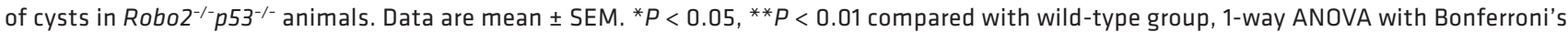
post hoc test (F). Scale bars: $10 \mu \mathrm{m}$ (C). (D) The cell differentiation defects in Robo2 ${ }^{-1-}$ kidneys were normalized in Robo2 and $p 53$-double knockout kidneys. Scale bars: $10 \mu \mathrm{m}$. (E and G) SA 3 -Gal staining indicated that double knockout of Robo2 and $p 53$ decreased senescence in Robo2 $2^{-1-} p 53^{-1-}$ kidneys compared with that in Robo2 $2^{-1-}$ kidneys and relative quantification. Asterisks in $\mathbf{E}$ indicate cysts. Data are mean $\pm \mathrm{SEM}$. ${ }^{* *} P<0.01$ compared with wild-type group, 1-way ANOVA with Bonferroni's post hoc test (G). Scale bars: $20 \mu \mathrm{m}(\mathbf{E}) .(n=3)$. 


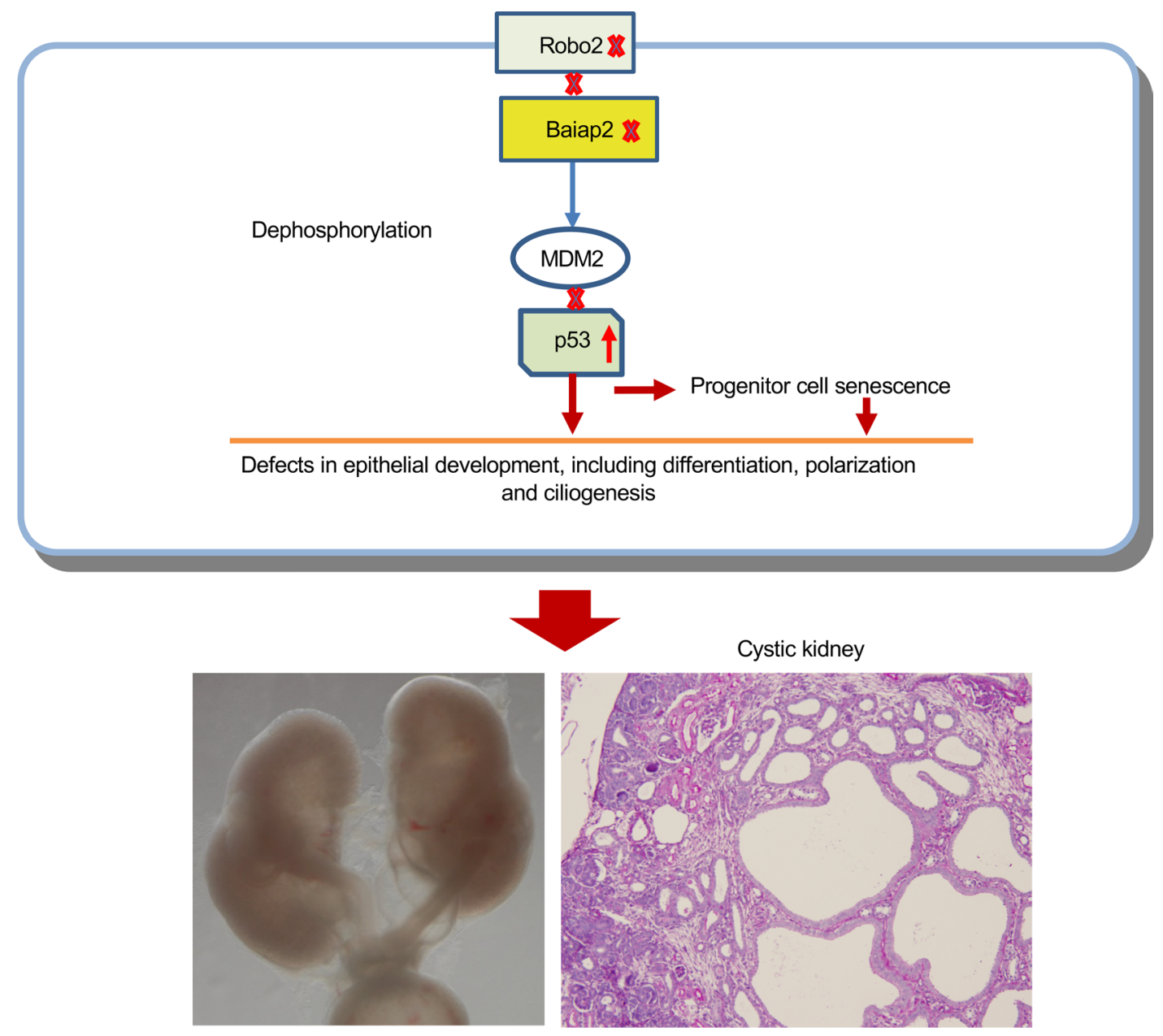

Figure 7. A schematic diagram depicting Robo2-Baiap2 integrated signaling in epithelial development. Robo2 deficiency led to high level of p53, resulted in progenitor cell senescence, and decreased the expression of ciliary and polarity proteins, which may contribute to differentiation defects and epithelial dysfunction.

the expression of PKC $\zeta, \mathrm{ZO}-2$, and Claudin-2 but also p21, CDK4, and SA $\beta-G a 1$. These results suggest that Robo2 regulates epithelial development by modulating Baiap2 signaling to p53 (Figure 7).

In summary, the present study provides evidence that Robo2-Baiap2 integrated signaling plays a crucial role in tubular epithelial cell development, including ciliogenesis, polarization, and differentiation of tubular epithelium. Robo2 deficiency caused cystic kidneys by defects of ciliogenesis, polarization, and differentiation of tubular epithelium. This conclusion is in line with observations in patients with renal cystic diseases $(1,2)$ and suggests a clinical opportunity to explore renal cystogenesis to target cystic disease.

\section{Methods}

Mice. B6.129S2-Trp53 ${ }^{\mathrm{tm} 1 \mathrm{Tyj}} / \mathrm{J}$ (stock 002101) mice were purchased from The Jackson Laboratory.

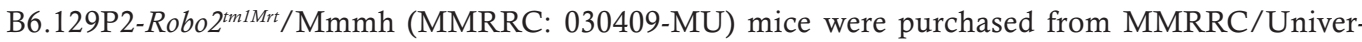
sity of Missouri.

Antibodies. The primary antibodies included anti-Robo2 (catalog sc-31607, Santa Cruz Biotechnology), anti-Robo2 (catalog MAB3147, R\&D Systems), fluorescein-conjugated LTL (catalog FL1321, Vector Labs), anti-phospho-histone H3 (Ser10) (catalog 9701S, Cell Signaling Technologies), rhodamine-conjugated phalloidin (R415, Invitrogen), anti-myc (9B11) mouse mAb (Sepharose Bead Conjugate, catalog 3400, Cell Signaling Technologies), anti-DYKDDDDK antibody (catalog 2368, Cell Signaling Technologies), anti-E-cadherin (catalog AF748, R\&D Systems), anti-Baiap2 (catalog 11087-2-AP, Proteintech), anti-E-cadherin (catalog ab53033, Abcam), anti-ZO-1 (catalog 617300, Invitrogen Zymed), anti-Myc (71D10) (catalog 2278, Cell Signaling Technologies), antilaminin (catalog L9393, MilliporeSigma), anti-PKC $\zeta$ (catalog sc-216, Santa Cruz Biotechnology), anti-CDK4 (H-22) (catalog sc-601, Santa Cruz Biotechnology), anti-Cited1 (catalog RB-9219, 
Neomarkers), anti-Claudin-2 (catalog 51-6100, Invitrogen Zymed), anti-Na ${ }^{+} / \mathrm{K}^{+}$-ATPase $\alpha-1$ subunit (catalog 05-369, MilliporeSigma), anti-GAPDH (catalog sc-32233, Santa Cruz Biotechnology), anti-Sox9 (catalog ab185966, Abcam), anti-Pax8 (catalog ab189249, Abcam), anti-p53 (catalog sc-126, Santa Cruz Biotechnology), Phospho-p53 Antibody Sampler Kit (catalog 9919, Cell Signaling Technologies), anti-p21 (F-5) (catalog sc-6246, Santa Cruz Biotechnology), anti-p21Cip1 (catalog ab2961, Abcam), anti-TRPV5 (catalog 17322-1AP, Proteintech), anti-ZONAB (catalog 402800, Invitrogen), anti-ZO-2 (catalog 38-9100, Invitrogen), and anti- $\alpha$-acetylated tubulin (catalog T6793, MilliporeSigma). The secondary antibodies included HRP-conjugated anti-goat, anti-mouse, and anti-rabbit antibodies (catalog A0181, A0216, and A0208, Beyotime); FITC-conjugated AffiniPure donkey anti-goat IgG $(\mathrm{H}+\mathrm{L})$ (Jackson ImmunoResearch); Cy3-conjugated AffiniPure donkey anti-goat IgG (H + L) (Jackson ImmunoResearch); FITC-conjugated AffiniPure donkey anti-rabbit IgG $(\mathrm{H}+\mathrm{L})$ (Jackson ImmunoResearch); and Cy3-conjugated AffiniPure donkey anti-rabbit IgG $(\mathrm{H}+\mathrm{L})$ (Jackson ImmunoResearch).

$S A \beta$-Gal. The SA $\beta$-Gal staining was performed on mouse kidney sections as previously described $(51,52)$. $\mathrm{SA} \beta-\mathrm{Gal}$ provides a simple assay for chromosomes that induce senescence. Briefly, kidney sections were fixed with 3.7\% paraformaldehyde in PBS and then incubated in staining solution ( $40 \mathrm{mM}$ citric acid/phosphate, $\mathrm{pH} 5.8,5 \mathrm{mM}$ potassium ferrocyanide, $5 \mathrm{mM}$ potassium ferricyanide, $150 \mathrm{mM} \mathrm{NaCl}, 2 \mathrm{mM} \mathrm{MgCl}, 1 \mathrm{mg} /$ $\mathrm{mL} \mathrm{X-gal)} \mathrm{at} 37^{\circ} \mathrm{C}$ for $16-18$ hours. Next, cells were washed twice with PBS, after which they were counterstained with $1 \%$ eosin solution for 5 minutes. Finally, blue cells in kidney sections were considered positive. To detect lysosomal $\beta$-Gal, the citric acid/sodium phosphate was at $\mathrm{pH} 4.0$. For each cortical region, 10 consecutive fields at original magnification $\times 200$ per section were selected for measurement (53). Signals were quantified by digital elaboration of microscopy images using ImageJ software (NIH, https://imagej.nih.gov/ij/).

Method of cilia length measurement. Murine IMCD3 cells were maintained at $37^{\circ} \mathrm{C}$ in $5 \% \mathrm{CO}_{2}$ in DMEM (Invitrogen) supplemented with 10\% FBS. To induce ciliary formation, cells were cultured in the serum-free medium for 48 or 72 hours. The RNA oligonucleotides that specifically targeted mouse Robo 2 were purchased from Santa Cruz Biotechnology. The RNA oligonucleotides were transfected with DharmaFECT siRNA transfection reagent (Dharmacon).

Cells were grown on glass coverslips and fixed with $95 \%$ cold methanol for 10 minutes at $-20^{\circ} \mathrm{C}$ followed by permeabilization with $0.2 \%$ Triton X-100 in PBS for 20 minutes. Cells were blocked with $2 \%$ bovine serum albumin in PBS for 1 hour. Cells were incubated with anti- $\alpha$-acetylated tubulin antibody (T6793, MilliporeSigma) for 1 hour at room temperature or overnight at $4^{\circ} \mathrm{C}$ and then with secondary antibody for 1 hour at room temperature. Cells were mounted with DAPI Fluoromount-G (Vectorlabs) and then examined with a Nikon Eclipse 80i microscope. The percentage of ciliated cells and the length of cilia were measured with the ImageJ software.

Immunofluorescent staining. The kidneys were fixed in 4\% paraformaldehyde (MilliporeSigma) overnight at $4^{\circ} \mathrm{C}$, and then the samples were equilibrated in $30 \%$ sucrose/PBS, $20 \%$ sucrose/PBS, and $10 \%$ sucrose/ PBS at $4^{\circ} \mathrm{C}$ for 16 hours, 6 hours, and 4 hours, respectively. The embryos were transferred to OCT embedding medium and mounted in OCT using cryomolds. The samples were sectioned ( $5 \mu \mathrm{m}$ thick) and permeabilized with $0.05 \%$ Triton X-100 and $0.1 \%$ SDS in PBS, pH 7.4. The samples were blocked with $0.1 \%$ casein (Vector Labs) and incubated overnight with the primary antibodies (diluted in $0.1 \%$ casein) at $4^{\circ} \mathrm{C}$. After extensive washing, the samples were incubated with a dilution of the appropriate fluorescent dye- or HRP-conjugated secondary antibody $(1: 400)$ at $4^{\circ} \mathrm{C}$ for 6 hours. The sections were counterstained with DAPI. The sections were then washed and mounted for observation, and each fluorescent channel was captured separately using a scanning confocal microscope (Olympus FluoView1000).

Microarray analysis and gene expression profiling. Microarray analysis was performed by the Microarray Core Facility at the CapitalBio Corporation. Affymetrix GeneChip Mouse Genome 4302.0 microarrays were used throughout the experiments (GEO GSE137298). The expression and functional profiles of genes were compared between the Robo2-null and wild-type kidneys. The functional profiling of the Robo2-regulated genes was performed using the Database for Annotation, Visualization, and Integrated Discovery based on the biological pathways from the Kyoto Encyclopedia of Genes and Genomes database, as previously described (54).

NanoString gene expression analysis. A total of 9 genes, including Cdkn1a, Cdkn1b, B-Raf, Cdc42, Ctnna1, Ctnnb1, Pten, Tjp1, and Trp53, were selected to analyze the expression of signature genes associated with the p53 pathway and TJ pathway. NanoString quantification of these genes' mRNA levels was performed using the nCounter analysis system and a custom-designed code set as previously described (see above). 
Two specific probes (capture and reporter) for each gene of interest were used. In brief, approximately 0.1-0.2 $\mu$ g of RNA was used to quantify the gene expression via the nCounter Analysis System according to the manufacturer's instructions. The mRNA molecules were counted on a NanoString nCounter as previously described $(55,56)$. The data analysis was performed according to the NanoString Technologies recommendations. The mRNA counts were processed to account for hybridization efficiency, background noise, and sample content. Each sample profile was normalized to the geometric mean of 3 housekeeping genes: Actb, B2m, and Pgk1 (Figure 2B).

Mass spectrometry-based IP. The embryonic kidney proteins were isolated to identify the endogenous Robo2-interacting proteins. The protein samples were prepared as previously described (57, 58). E15 embryonic kidney proteins were isolated and extracted in ice-cold RIPA lysis and extraction buffer (50 mM Tris, pH 7.4; $150 \mathrm{mM} \mathrm{NaCl} ; 0.1 \%$ SDS; $1 \%$ NP-40; 0.5\% sodium deoxycholate; $1 \mathrm{mM}$ $\mathrm{Na}_{3} \mathrm{VO}_{4} ; 1 \times$ protease inhibitor; and $1 \mathrm{mM} \mathrm{NaF}$ ). After preclearing the lysate, $2 \mu \mathrm{g}$ of the Robo 2 antibody was mixed with $1 \mathrm{mg}$ of lysate and $80 \mu \mathrm{L}$ of Protein A/G agarose beads (sc-2003, Santa Cruz Biotechnology) and incubated overnight at $4^{\circ} \mathrm{C}$ to immunoprecipitate the proteins. The beads were washed 3 times with ice-cold washing buffer and resuspended in SDS loading buffer (Thermo Scientific Pierce). The samples were loaded onto an SDS-PAGE gel. The entire gel was cut for each run and divided into 4 sections according to molecular size. The cut gel was subjected to in-gel digestion, and then 2D LC/MS analysis was performed as previously described $(57,58)$. The data were further analyzed by the ProteinLynx Global Server version 2.5 (Waters Corporation).

$R N A$ interference. The custom and chemically modified siRNAs were designed to target Robo2 (GenePharma), 5'-GGCCAUGAGUACUGAUGAATT-3', 3'-UUCAUCGUACUCAUGGCCTT-5'; Baiap2 (GenePharma), 5'-GCACUGAAGAAAUACCAAATT-3'; Tjp1 (GenePharma), 5'-GGAAACAGCUAUAUGGGAATT-3', 3'-UUCCCAUAUAGCUGUUUCCTT-5'; and Ybx-3 (GenePharma), 5'-CCGACGUCCCUACAACUAUTT-3'， 3'-AUAGUUGUAGGGACGUCGGTT-5'. A nonspecific siRNA duplex served as a control (GenePharma). To knock down Robo2, Baiap2, Tjp1, Trp53, ZONAB, and $Y b x-3$ in vitro, the MDCK cells were transfected with the individual siRNAs using the JetPRIME Reagent (POLYPUS, 114-75).

To assess the effects of Slit 2 and of silencing of Robo2 on gene expression, we starved MDCK cells overnight in DMEM/F-12 (Invitrogen) supplemented with 0.1\% FBS, and cells were treated with ROBO2 siRNAs and then with $50 \mathrm{nM}$ recombinant human SLIT2 N-terminus (MilliporeSigma, SRP3155) or buffer for 24 hours as previously described $(59,60)$. After the media were removed, cells were lysed for Western blotting.

Co-IP. To test whether Robo2 and Baiap2 interacted in vitro, we designed constructs of Robo2 and Baiap2 (a gift from Naoki Mochizuki, Department of Cell Biology, National Cerebral and Cardiovascular Center Research Institute, Osaka, Japan). Ten percent of the total lysate was reserved; the remaining was centrifuged and the supernatant was transferred to a clean microcentrifuge tube (Thermo Fisher Scientific). The lysate was precleared by incubation with Protein A/G agarose for 3 hours on a rotator at $4^{\circ} \mathrm{C}$. The beads were centrifuged, and the supernatants were transferred to fresh tubes, where they were incubated with $5 \mu \mathrm{L}$ Robo2 antibody for 1 hour before $50 \mu \mathrm{L}$ of a homogeneous Protein A/G agarose suspension was added. The samples were then incubated overnight at $4^{\circ} \mathrm{C}$ on a rotator. The complexes were centrifuged, the supernatant was removed, and the beads were washed twice with lysis buffer. Protein sample buffer was added and the samples were boiled for 3 minutes. The complexes were then analyzed as described in the immunoblotting section.

Pull-down assay. GST fusion proteins were purified as formerly described (61). For pull-down studies, purified full-length Baiap2-GST, Baiap2-250-GST, or Baiap2-1-250-GST fusion proteins from E. coli Rosetta (DE3) were incubated with glutathione-agarose beads in $\mathrm{PBS}$ buffer $(\mathrm{pH} 7.4)$ at $4^{\circ} \mathrm{C}$ with rotation and washed repeatedly with $\mathrm{PBS}$ buffer ( $\mathrm{pH}$ 7.4). When appropriate, the beads were incubated with reconstituted Robo2-His or Robo2-CC3-3-His from E. coli Rosetta (DE3) overnight at $4^{\circ} \mathrm{C}$, followed by a wash step in PBS buffer ( $\mathrm{pH}$ 7.4). Bound proteins were collected by centrifugation and resolved by SDS-PAGE. Western blots used anti-GST (M20007, Abmart) and anti-HIS (M20001, Abmart)

Immunoblotting. The Western blots were performed as previously described $(62,63)$. The isolated kidneys were washed with ice-cold PBS, $\mathrm{pH} 7.4$, and lysed with ice-cold RIPA lysis buffer at $4^{\circ} \mathrm{C}$ for 1 hour. The samples were centrifuged at $12,000 \mathrm{~g}$ for 15 minutes at $4^{\circ} \mathrm{C}$. The samples were subjected to SDS-PAGE, transferred to PVDF membranes, and detected with the appropriate primary antibodies followed by HRP-conjugated antimouse, -goat, or -rabbit IgGs. The blotting signals were detected by enhanced chemiluminescence using the SuperSignal West Dura Extended Duration Substrate (Pierce). 
Notably, we routinely isolated proteins from the C57BL/6 wild-type and Robo2-mutant kidneys, as well as the cultured HEK and MDCK cells. However, the amounts of the total isolated proteins were adjusted to obtain identical quantities and used as the starting materials for comparisons. The primary and secondary antibodies were used as described above. Bands were detected by enhanced chemiluminescence, and the relative protein expression level was quantified using ImageJ.

Tubular dilation/cystic index. To quantify the rate of cystic kidneys, we applied a grid of squares 13.625 $\mu \mathrm{m}$ large to sections of kidneys stained with H\&E. We marked each cross with a dot, and we counted the number of dots inside the lumen of 3 litters. We determined the degree of dilatation according to criteria previously established $(64,65)$. Briefly, 1 dot: normal tubules; 2 dots: dilated tubules; $>3$ dots: cysts.

Data analysis. All experiments were performed at least 3 times $(n)$ and the values presented as mean \pm SEM. Statistical significance was determined using the 2-tailed Student's $t$ test. Probability $(P)$ values are indicated with * and **, which correspond to values of 0.05 and 0.01 , respectively. For all statistical comparisons, $P$ values less than 0.05 were accepted to indicate significant differences.

Study approval. All animal procedures were approved by the Institutional Animal Care and Use Committee at the Chinese PLA General Hospital and Medical School of Chinese PLA.

\section{Author contributions}

QL, YX, XL, and XC conceived and designed the research. QL, SC, QM, YL, HY, GG, EA, CR, KW, YZ, and XB performed experiments. GG and QL analyzed data. JY, XB, YX, XL, and XC interpreted results of experiments. QL and GC prepared figures. QL and XL drafted the manuscript. QL, YX, XL, and XC edited and revised the manuscript. QL, SC, QM, YL, HY, GG, EA, CR, KW, YZ, JY, XB, GC, YX, XL, and $\mathrm{XC}$ approved the final version of the manuscript.

\section{Acknowledgments}

We thank Fujian Zhang, Wanjun Shen, and Guanyong Chen for critical discussion. This study was supported by funds from National Key Research and Development Program of China (2016YFC1101403 and 2016YFC0901502), National Natural Science Foundation of China (81170631, 81570597, and 81330019), and Basic and Frontier Research Program of Chongqing (cstc2017jcyjBX0014). XL acknowledges support from NIH grants R01 DK084097 and P30 DK106912.

Address correspondence to: Yuansheng Xie or Xiangmei Chen, Department of Nephrology, Chinese PLA General Hospital, 28 Fuxing Road, Haidian District, Beijing, 100853, China. Phone: 8610.66935462; Email: xieyuansn@aliyun.com (YX); xmchen301@126.com (XC). Or to: Xiaogang Li, Department of Internal Medicine, Mayo Clinic, Stabile 7-52, 200 1st St. SW, Rochester, MN 55905, USA. Phone: 507.752.0110; 1i.xiaogang@mayo.edu.

1. Cornec-Le Gall E, Audrézet MP, Le Meur Y, Chen JM, Férec C. Genetics and pathogenesis of autosomal dominant polycystic kidney disease: 20 years on. Hum Mutat. 2014;35(12):1393-1406.

2. Kagan KO, Dufke A, Gembruch U. Renal cystic disease and associated ciliopathies. Curr Opin Obstet Gynecol. 2017;29(2):85-94.

3. Charrier LE, Loie E, Laprise P. Mouse Crumbs3 sustains epithelial tissue morphogenesis in vivo. Sci Rep. 2015;5:17699.

4. Cereijido M, Contreras RG, Shoshani L, Flores-Benitez D, Larre I. Tight junction and polarity interaction in the transporting epithelial phenotype. Biochim Biophys Acta. 2008;1778(3):770-793.

5. Dressler GR. Polarity and renal cystogenesis. J Am Soc Nephrol. 2012;23(1):4-5.

6. Seeger M, Tear G, Ferres-Marco D, Goodman CS. Mutations affecting growth cone guidance in Drosophila: genes necessary for guidance toward or away from the midline. Neuron. 1993;10(3):409-426.

7. Rothberg JM, Hartley DA, Walther Z, Artavanis-Tsakonas S. slit: an EGF-homologous locus of D. melanogaster involved in the development of the embryonic central nervous system. Cell. 1988;55(6):1047-1059.

8. Hwang DY, et al. Mutations of the SLIT2-ROBO2 pathway genes SLIT2 and SRGAP1 confer risk for congenital anomalies of the kidney and urinary tract. Hum Genet. 2015;134(8):905-916.

9. Grieshammer U, Le Ma, Plump AS, Wang F, Tessier-Lavigne M, Martin GR. SLIT2-mediated ROBO2 signaling restricts kidney induction to a single site. Dev Cell. 2004;6(5):709-717.

10. Hwang DY, et al. Mutations in 12 known dominant disease-causing genes clarify many congenital anomalies of the kidney and urinary tract. Kidney Int. 2014;85(6):1429-1433.

11. $\mathrm{Lu} \mathrm{W}$, et al. Disruption of ROBO2 is associated with urinary tract anomalies and confers risk of vesicoureteral reflux. Am $J$ Hum Genet. 2007;80(4):616-632.

12. Dobson MG, Darlow JM, Hunziker M, Green AJ, Barton DE, Puri P. Heterozygous non-synonymous ROBO2 variants are unlikely to be sufficient to cause familial vesicoureteric reflux. Kidney Int. 2013;84(2):327-337. 
13. Morizane R, Lam AQ, Freedman BS, Kishi S, Valerius MT, Bonventre JV. Nephron organoids derived from human pluripotent stem cells model kidney development and injury. Nat Biotechnol. 2015;33(11):1193-1200.

14. Narlis M, Grote D, Gaitan Y, Boualia SK, Bouchard M. Pax2 and pax8 regulate branching morphogenesis and nephron differentiation in the developing kidney. J Am Soc Nephrol. 2007;18(4):1121-1129.

15. Torban E, Goodyer P. What PAX genes do in the kidney. Exp Nephrol. 1998;6(1):7-11.

16. Murphy AJ, et al. SIX2 and CITED1, markers of nephronic progenitor self-renewal, remain active in primitive elements of Wilms' tumor. J Pediatr Surg. 2012;47(6):1239-1249.

17. Brown AC, Muthukrishnan SD, Oxburgh L. A synthetic niche for nephron progenitor cells. Dev Cell. 2015;34(2):229-241.

18. Little MH, Kairath P. Does renal repair recapitulate kidney development? J Am Soc Nephrol. 2017;28(1):34-46.

19. Puri P, et al. Ectopic phosphorylated Creb marks dedifferentiated proximal tubules in cystic kidney disease. Am J Pathol. 2018;188(1):84-94.

20. Kumar S, et al. Sox 9 activation highlights a cellular pathway of renal repair in the acutely injured mammalian kidney. Cell Rep 2015;12(8):1325-1338

21. Kang HM, Huang S, Reidy K, Han SH, Chinga F, Susztak K. Sox9-positive progenitor cells play a key role in renal tubule epithelial regeneration in mice. Cell Rep. 2016;14(4):861-871.

22. Mazal PR, et al. Derivation of nephrogenic adenomas from renal tubular cells in kidney-transplant recipients. $N$ Engl JMed. 2002;347(9):653-659.

23. Fan X, et al. Inhibitory effects of Robo2 on nephrin: a crosstalk between positive and negative signals regulating podocyte structure. Cell Rep. 2012;2(1):52-61.

24. Kast DJ, et al. Mechanism of IRSp53 inhibition and combinatorial activation by Cdc42 and downstream effectors. Nat Struct Mol Biol. 2014;21(4):413-422.

25. Crespi A, et al. LIN7 regulates the filopodium- and neurite-promoting activity of IRSp53. J Cell Sci. 2012;125(pt 19):4543-4554

26. Distefano G, et al. Polycystin-1 regulates extracellular signal-regulated kinase-dependent phosphorylation of tuberin to control cell size through mTOR and its downstream effectors S6K and 4EBP1. Mol Cell Biol. 2009;29(9):2359-2371.

27. Massari S, et al. LIN7 mediates the recruitment of IRSp53 to tight junctions. Traffic. 2009;10(2):246-257.

28. Lima WR, et al. ZONAB promotes proliferation and represses differentiation of proximal tubule epithelial cells. $J A m$ Soc Nephrol. 2010;21(3):478-488.

29. Krenning L, Feringa FM, Shaltiel IA, van den Berg J, Medema RH. Transient activation of p53 in G2 phase is sufficient to induce senescence. Mol Cell. 2014;55(1):59-72.

30. Muñoz-Espín D, et al. Programmed cell senescence during mammalian embryonic development. Cell. 2013;155(5):1104-1118.

31. Purvis JE, Karhohs KW, Mock C, Batchelor E, Loewer A, Lahav G. p53 dynamics control cell fate. Science. 2012;336(6087):1440-1444.

32. Ruan YC, et al. CFTR interacts with ZO-1 to regulate tight junction assembly and epithelial differentiation through the ZONAB pathway. J Cell Sci. 2014;127(pt 20):4396-4408.

33. Sedelnikova OA, Horikawa I, Zimonjic DB, Popescu NC, Bonner WM, Barrett JC. Senescing human cells and ageing mice accumulate DNA lesions with unrepairable double-strand breaks. Nat Cell Biol. 2004;6(2):168-170.

34. Harburg G, et al. SLIT/ROBO2 signaling promotes mammary stem cell senescence by inhibiting Wnt signaling. Stem Cell Reports. 2014;3(3):385-393.

35. Meek DW, Knippschild U. Posttranslational modification of MDM2. Mol Cancer Res. 2003;1(14):1017-1026.

36. Marine JC, Lozano G. Mdm2-mediated ubiquitylation: p53 and beyond. Cell Death Differ. 2010;17(1):93-102.

37. Umeda $\mathrm{K}$, et al. ZO-1 and ZO-2 independently determine where claudins are polymerized in tight-junction strand formation. Cell. 2006;126(4):741-754.

38. Pozzi A, Zent R. ZO-1 and ZONAB interact to regulate proximal tubular cell differentiation. J Am Soc Nephrol. 2010;21(3):388-390.

39. Odenwald MA, et al. ZO-1 interactions with F-actin and occludin direct epithelial polarization and single lumen specification in 3D culture. J Cell Sci. 2017;130(1):243-259.

40. Castelli M, et al. Polycystin-1 binds Par3/aPKC and controls convergent extension during renal tubular morphogenesis. Nat Commun. 2013;4:2658.

41. Larre I, et al. Ouabain modulates ciliogenesis in epithelial cells. Proc Natl Acad Sci U S A. 2011;108(51):20591-20596

42. Disanza A, et al. Regulation of cell shape by Cdc42 is mediated by the synergic actin-bundling activity of the Eps8-IRSp53 complex. Nat Cell Biol. 2006;8(12):1337-1347.

43. Shieh SY, Ikeda M, Taya Y, Prives C. DNA damage-induced phosphorylation of p53 alleviates inhibition by MDM2. Cell. 1997;91(3):325-334.

44. Mayo LD, Donner DB. A phosphatidylinositol 3-kinase/Akt pathway promotes translocation of Mdm2 from the cytoplasm to the nucleus. Proc Natl Acad Sci U S A. 2001;98(20):11598-11603.

45. Zhou BP, Liao Y, Xia W, Zou Y, Spohn B, Hung MC. HER-2/neu induces p53 ubiquitination via Akt-mediated MDM2 phosphorylation. Nat Cell Biol. 2001;3(11):973-982.

46. Godley LA, Kopp JB, Eckhaus M, Paglino JJ, Owens J, Varmus HE. Wild-type p53 transgenic mice exhibit altered differentiation of the ureteric bud and possess small kidneys. Genes Dev. 1996;10(7):836-850.

47. Hilliard S, Aboudehen K, Yao X, El-Dahr SS. Tight regulation of p53 activity by Mdm2 is required for ureteric bud growth and branching. Dev Biol. 2011;353(2):354-366.

48. Zhou X, Fan LX, Sweeney WE, Denu JM, Avner ED, Li X. Sirtuin 1 inhibition delays cyst formation in autosomal-dominant polycystic kidney disease. J Clin Invest. 2013;123(7):3084-3098.

49. Gibson MC, Perrimon N. Apicobasal polarization: epithelial form and function. Curr Opin Cell Biol. 2003;15(6):747-752.

50. Nagaoka K, Udagawa T, Richter JD. CPEB-mediated ZO-1 mRNA localization is required for epithelial tight-junction assembly and cell polarity. Nat Commun. 2012;3:675.

51. Gutiérrez-Fernández A, et al. Loss of MT1-MMP causes cell senescence and nuclear defects which can be reversed by retinoic acid. EMBO J. 2015;34(14):1875-1888.

52. Yang HH, et al. Inhibitory effects of juglanin on cellular senescence in human dermal fibroblasts. J Nat Med. 2014;68(3):473-480. 
53. Jin H, et al. Epithelial innate immunity mediates tubular cell senescence after kidney injury. JCI Insight. 2019;4(2):125490.

54. Li X, et al. Aiolos promotes anchorage independence by silencing p66Shc transcription in cancer cells. Cancer Cell. 2014;25(5):575-589

55. Veldman-Jones $\mathrm{MH}$, et al. Evaluating robustness and sensitivity of the nanostring technologies ncounter platform to enable multiplexed gene expression analysis of clinical samples. Cancer Res. 2015;75(13):2587-2593.

56. Geiss GK, et al. Direct multiplexed measurement of gene expression with color-coded probe pairs. Nat Biotechnol. 2008;26(3):317-325.

57. Marcon E, et al. Assessment of a method to characterize antibody selectivity and specificity for use in immunoprecipitation. Nat Methods. 2015;12(8):725-731.

58. Vizoso M, et al. Epigenetic activation of a cryptic TBC1D16 transcript enhances melanoma progression by targeting EGFR Nat Med. 2015;21(7):741-750.

59. Rama N, et al. Slit2 signaling through Robo1 and Robo2 is required for retinal neovascularization. Nat Med. 2015;21(5):483-491.

60. Fan X, et al. SLIT2/ROBO2 signaling pathway inhibits nonmuscle myosin IIA activity and destabilizes kidney podocyte adhesion. JCI Insight. 2016;1(19):e86934.

61. Harper S, Speicher DW. Purification of proteins fused to glutathione S-transferase. Methods Mol Biol. 2011;681:259-280.

62. Hayashi MT, Cesare AJ, Fitzpatrick JA, Lazzerini-Denchi E, Karlseder J. A telomere-dependent DNA damage checkpoint induced by prolonged mitotic arrest. Nat Struct Mol Biol. 2012;19(4):387-394.

63. Zhou WJ, Geng ZH, Spence JR, Geng JG. Induction of intestinal stem cells by R-spondin 1 and Slit2 augments chemoradioprotection. Nature. 2013;501(7465):107-111.

64. Bastos AP, et al. Pkd1 haploinsufficiency increases renal damage and induces microcyst formation following ischemia/reperfusion. J Am Soc Nephrol. 2009;20(11):2389-2402.

65. Rowe I, et al. Defective glucose metabolism in polycystic kidney disease identifies a new therapeutic strategy. Nat Med. $2013 ; 19(4): 488-493$ 